ALEA, Lat. Am. J. Probab. Math. Stat. 19, 467-492 (2022)

DOI: 10.30757/ALEA.v19-18

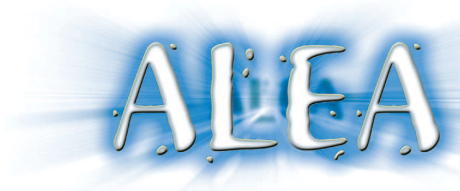

\title{
A Markov process for an infinite age-structured population
}

\section{Dominika Jasińska and Yuri Kozitsky ${ }^{1}$}

Instytut Matematyki, Uniwersytet Marii Curie-Skłodowskiej, plac Marii Curie-Skłodwskiej 5, 20-031 Lublin, Poland

E-mail address: jasdominika@wp.pl

Instytut Matematyki, Uniwersytet Marii Curie-Skłodowskiej, plac Marii Curie-Skłodwskiej 5, 20-031 Lublin, Poland

E-mail address: jkozi@hektor.umcs.lublin.pl

\begin{abstract}
A Markov process is constructed in an explicit way for an infinite system of entities arriving in and departing from a habitat $X$, which is a locally compact Polish space with a positive Radon measure $\chi$. Along with its location $x \in X$, each particle is characterized by age $\alpha \geq 0$ - time since arriving. As the state space one takes the set of marked configurations $\widehat{\Gamma}$, equipped with a metric that makes it a complete and separable metric space. The stochastic evolution of the system is described by a Kolmogorov operator $L$, expressed through the measure $\chi$ and a departure rate $m(x, \alpha) \geq 0$, and acting on bounded continuous functions $F: \widehat{\Gamma} \rightarrow \mathbb{R}$. For this operator, we pose the martingale problem and show that it has a unique solution, explicitly constructed in the paper. We also prove that the corresponding process has a unique stationary state and is temporarily egrodic if the rate of departure is separated away from zero.
\end{abstract}

\section{Introduction}

In recent years, the stochastic dynamics of age-structured populations attract considerable attention, mostly due to their various applications, see He et al. (2019); Jagers and Klebaner (2000); Kozitsky (2021); Méléard and Tran (2012); Wang (2019). Finite populations of this kind are much more mathematically tractable, in contrast to infinite ones where only few results are known. In the present work, we construct a Markov process for an infinite system of point entities that arrive in and depart from a locally compact habitat.

Let $X$ be a locally compact Polish space and $\chi$ a positive Radon measure defined thereon. $X$ will serve as the habitat for infinite populations of entities (particles) that arrive in and depart from $X$ at random. Along with the location $x \in X$, each entity is characterized by age $\alpha \in[0+\infty)=: \mathbb{R}_{+}$ - time of its presence in the population. The probability that an entity appears at time $t$ in a given compact $\Lambda \subset X$ is set to be $1-\exp (-t \chi(\Lambda))$. Similarly, the probability that the entity located at $x$ departs at time $t$ is $1-\exp (-\operatorname{tm}(x, \alpha))$, where $\alpha$ is its age at the moment of departure. Since we do not assume that $\chi(X)$ be finite, the total population can get instantly infinite, even if it is finite

\footnotetext{
${ }^{1}$ Corresponding author

Received by the editors July 9th, 2020; accepted November 16th, 2021.

2010 Mathematics Subject Classification. 60J25; 60J85; 92D25.

Key words and phrases. Age-structured population; Fokker-Planck equation; martingale problem; Markov processes.
} 
in the initial state. That is, our model is characterized by an arriving measure $\chi$ and a departing rate $m(x, \alpha)$.

To describe microscopic states of the population, we use so called marked configurations $\hat{\gamma}$, cf. Definition 9.1.II, page 3 of Daley and Vere-Jones (2008). To define them, we set

$$
\Gamma=\{\gamma \subset X:|\gamma \cap \Lambda|<\infty, \text { for each compact } \Lambda \subset X\}
$$

where $|\gamma \cap \Lambda|$ is the number of the elements of $\gamma$ contained in $\Lambda$. Let $\mathcal{A}$ be the set of finite configurations $a=\left\{\alpha_{1}, \ldots, \alpha_{n}\right\} \subset[0,+\infty), n \in \mathbb{N}_{0}$. This set - equipped with a metric that makes it a complete and locally compact metric space - will serve as the space of marks for our model. That is, for $x \in X$, by $a(x) \in \mathcal{A}$ we mean the ages of particles located at $x$. Then a marked configuration $\hat{\gamma}$ is the pair $(\gamma, a)$ with $\gamma \in \Gamma$ and $a: X \rightarrow \mathcal{A}$. In this case, we also write $\gamma=p(\hat{\gamma})$ and call $\gamma$ ground configuration for $\hat{\gamma}$. The set of all marked configurations $\widehat{\Gamma}$ is equipped with a metric, in which it is complete and separable. Elements of $\hat{\gamma}$ are pairs $(x, \alpha)=: \hat{x}$. They can be enumerated in $\hat{\gamma}$, which allows one to consider sums $\sum_{\hat{x} \in \hat{\gamma}} g(\hat{x})$ for continuous compactly supported functions $g(\hat{x})=g(x, \alpha)$.

The evolution of the population is governed by the Kolmogorov equation

$$
\frac{d}{d t} F_{t}=L F_{t},\left.\quad F_{t}\right|_{t=0}=F_{0},
$$

where $t$ is time, $F_{t}$ is a suitable (test) function of $\hat{\gamma}$ and the Kolmogorov operator $L$ is given by the following expression

$$
\begin{array}{r}
(L F)(\hat{\gamma})=\sum_{(x, \alpha) \in \hat{\gamma}} \frac{\partial}{\partial \alpha} F(\hat{\gamma})+\sum_{(x, \alpha) \in \hat{\gamma}} m(x, \alpha)[F(\hat{\gamma} \backslash(x, \alpha))-F(\hat{\gamma})] \\
+\int_{X}[F(\hat{\gamma} \cup(x, 0))-F(\hat{\gamma})] \chi(d x) .
\end{array}
$$

Here the first term describes aging, the second one corresponds to departing, whereas the last term describes the appearance of new entities. In (1.2) and and in the sequel, in expressions like $\hat{\gamma} \cup \hat{x}$ we consider $\hat{x}=(x, \alpha)$ as a singleton configuration. The model parameters are subject to the following assumptions:

(i) The departure rate $X \times \mathbb{R}_{+} \ni(x, \alpha) \mapsto m(x, \alpha) \in \mathbb{R}_{+}$is continuous and bounded, i.e., such that $m(x, \alpha) \leq m_{*}$ for some $m_{*}>0$ and all $(x, \alpha)$. Moreover, there exits $\varkappa:[0,1] \rightarrow \mathbb{R}_{+}$ such that $\varkappa(\epsilon) \rightarrow 0$ as $\epsilon \rightarrow 0$ and the following holds

$$
\forall x \in X \quad\left|m(x, \alpha)-m\left(x, \alpha^{\prime}\right)\right| \leq \varkappa\left(\left|\alpha-\alpha^{\prime}\right|\right), \quad\left|\alpha-\alpha^{\prime}\right| \in(0,1) .
$$

(ii) The arriving measure $\chi$ is just a positive Radon measure.

The result of the present work can be outlined as follows. We introduce a Banach space $\mathcal{C}$ of bounded continuous functions $F: \widehat{\Gamma} \rightarrow \mathbb{R}$, in which we define $L$ as a closed and densely defined linear operator that satisfies the conditions of the Hille-Yosida theorem, and hence is the generator of a $C_{0}$-semigroup $\{S(t)\}_{t \geq 0}$. Then the solution of (1.1) is obtained in the form $F_{t}=S(t) F_{0}$. For a class of functions $\mathcal{F}_{\Theta}, F_{t}$ corresponding to $F_{0} \in \mathcal{F}_{\Theta}$ is obtained in an explicit way. This allows us to explicitly construct the corresponding Markov transition function $p_{t}^{\hat{\gamma}}$ and obtain finite-dimensional laws of a Markov process $\mathcal{X}$ with values in $\widehat{\Gamma}$, which describes the stochastic evolution of our model. Possible objects of this kind are specified as stochastic processes that solve the martingale problem for $L$. Then we show that this problem is well-posed, i.e., uniqueness holds. The main ingredient of the proof here is showing that the corresponding Fokker-Planck equation for $L$ has a unique solution, which we do by employing the resolvent of $L$. Assuming that $m(x, \alpha) \geq m_{0}>0$, we also show that the process $\mathcal{X}$ has a unique stationary state, explicitly constructed in the paper, such that the laws of $\mathcal{X}(t)$ weakly converge to this state as $t \rightarrow+\infty$. 


\section{Preliminaries}

In this work, we use the following standard notions and notations. For a Polish (=separable and completely metrizable) space $E$, by $\mathcal{B}(E)$ we denote the corresponding Borel $\sigma$-field; $C_{\mathrm{b}}(E)$ (resp. $\left.B_{\mathrm{b}}(E)\right)$ stands for the set of all bounded and continuous (resp. bounded and measurable) functions $f: E \rightarrow \mathbb{R}$. By $C_{\mathrm{b}}^{+}(E)$ we denote the set of positive elements of $C_{\mathrm{b}}(E)$. A subset $C_{\mathrm{cs}}(E) \subset C_{\mathrm{b}}(E)$ consists of continuous compactly supported functions. A family of functions $\mathcal{F}$ is said to separate the points of $E$ if for each distinct $x, y \in E$, one finds $f \in \mathcal{F}$ such that $f(x) \neq f(y)$. By $\sigma \mathcal{F}$ we denote the smallest sub-field of $\mathcal{B}(E)$ such that each $f \in \mathcal{F}$ is $\sigma \mathcal{F}$-measurable; by $\mathcal{P}(E)$ we mean the set of all probability Borel measures on $(E, \mathcal{B}(E))$. For a given measure $\mu$ and a suitable function $f$, we write $\mu(f)=\int f d \mu$. For a sequence $\left\{\mu_{n}\right\}_{n \in \mathbb{N}} \subset \mathcal{P}(E)$, by writing $\mu_{n} \Rightarrow \mu \in \mathcal{P}(E)$ we mean its weak convergence, i.e., $\mu_{n}(f) \rightarrow \mu(f)$ for all $f \in C_{\mathrm{b}}(E)$. A family of functions $\mathcal{F}$ is said to be separating if $\mu_{1}(f)=\mu_{2}(f)$ holding for all $f \in \mathcal{F}$ implies $\mu_{1}=\mu_{2}$ for any $\mu_{1}, \mu_{2} \in \mathcal{P}(E)$. If $\mathcal{F}$ separates the points of $E$ and its linear span is an algebra with respect to pointwise operations, then it is separating, see Ethier and Kurtz (1986), Theorem 4.5 on page 113. A family of functions $\mathcal{F}$ is said to be convergence determining if $\mu_{n}(f) \rightarrow \mu(f)$ holding for all $f \in \mathcal{F}$ implies $\mu_{n} \Rightarrow \mu$. For a suitable $A \subset E$, by $\mathbb{1}_{A}$ we denote the indicator of $A$, i.e., the functions such that $\mathbb{1}_{A}(x)=1$ if $x \in A$ and $\mathbb{1}_{A}(x)=0$ otherwise.

2.1. The space of marks. As mentioned above, our populations dwell in a locally compact Polish space $X$. By $\Lambda$ we always denote a compact subset of $X$. Each population member is characterized by its compound trait $\hat{x}=(x, \alpha) \in \widehat{X}=X \times \mathbb{R}_{+}$. For a function, $g: \widehat{X} \rightarrow \mathbb{R}$, we use interchangeable writings $g(\hat{x})$ and $g(x, \alpha)$. The space $\widehat{X}$ is equipped with the product topology assuming that the topology of $\mathbb{R}_{+}$be defined by the metric which we introduce now. For $\alpha \geq 0$, we set $\omega(\alpha)=$ $\min \{\alpha ; 1 / \alpha\}$, and then

$$
r(\alpha, 0)=\omega(\alpha), \quad r\left(\alpha, \alpha^{\prime}\right)=\min \left\{\left|\alpha-\alpha^{\prime}\right| ; \omega(\alpha)+\omega\left(\alpha^{\prime}\right)\right\},
$$

where $|\beta|$ is the usual absolute value of $\beta \in \mathbb{R}$.

Proposition 2.1. The above introduced $r$ is a metric such that $\left(\mathbb{R}_{+}, r\right)$ be a compact metric space.

Proof: To prove the first part we just have to check the validity of the triangle inequality

$$
r\left(\alpha_{1}, \alpha_{2}\right) \leq r\left(\alpha_{1}, \alpha_{3}\right)+r\left(\alpha_{2}, \alpha_{3}\right) .
$$

This technical exercise is made in Appendix. To prove the compactness, we have to show that: (a) $r$ is complete; (b) the space $\left(\mathbb{R}_{+}, r\right)$ is totally bounded. Let $\left\{\alpha_{n}\right\}_{n \in \mathbb{N}} \subset \mathbb{R}_{+}$be an $r$-Cauchy sequence. Here one may have the following possibilities: (i) there exists $\bar{\alpha}<\infty$ such that $\alpha_{n} \leq \bar{\alpha}$, $n \in \mathbb{N}$; (ii) the considered sequence contains a subsequence that diverges in the usual sense. In case (i), $\left\{\alpha_{n}\right\}_{n \in \mathbb{N}}$ contains a subsequence, say $\left\{\alpha_{n_{k}}\right\}_{k \in \mathbb{N}}$, such that $\left|\alpha_{n_{k}}-\alpha_{*}\right| \rightarrow 0$ as $k \rightarrow+\infty$ for some $\alpha_{*} \leq \bar{\alpha}$. At the same time, for $\varepsilon<2 / \bar{\alpha}, r\left(\alpha_{n}, \alpha_{m}\right)<\varepsilon$ implies $\left|\alpha_{n}-\alpha_{m}\right|<\varepsilon$, see (2.1), which means that $r\left(\alpha_{n}, \alpha_{*}\right) \rightarrow 0$ as $n \rightarrow+\infty$. In case (ii), the divergent subsequence converges in $r$ to zero, which implies that the whole sequence converges to zero in $r$. Hence, the latter metric is complete. To prove (b), we set $\tilde{B}_{\varepsilon}(\alpha)=\left\{\alpha^{\prime} \in \mathbb{R}_{+}: r\left(\alpha, \alpha^{\prime}\right)<\varepsilon\right\}$. Fix $\varepsilon \in(0,1)$ and take the least $k \in \mathbb{N}$ such that $k+1>1 / \varepsilon^{2}$. Then $\mathbb{R}_{+}=\cup_{j=0}^{k} \tilde{B}_{\varepsilon}(j \varepsilon)$, which yields the property in question.

Let us now compare $r$ with the absolute-value metrics of $\mathbb{R}_{+}$. By $C\left(\mathbb{R}_{+}, \mathcal{T}_{r}\right)$ we will mean the set of all bounded $r$-continuous functions, whereas $C_{\mathrm{b}}\left(\mathbb{R}_{+}, \mathcal{T}_{|\cdot|}\right)$ is going to stand for the set of all bounded $|\cdot|$-continuous functions.

Proposition 2.2. $\mathcal{T}_{r}$ is coarser than $\mathcal{T}_{|\cdot|}$, and hence the embedding $\left(\mathbb{R}_{+}, \mathcal{T}_{|\cdot|}\right) \hookrightarrow\left(\mathbb{R}_{+}, \mathcal{T}_{r}\right)$ is continuous, whereas both latter topological spaces are Borel isomorphic. Moreover,

$$
C\left(\mathbb{R}_{+}, \mathcal{T}_{r}\right)=\left\{u \in C_{\mathrm{b}}\left(\mathbb{R}_{+}, \mathcal{T}_{|\cdot|}\right): \lim _{\alpha \rightarrow+\infty} u(\alpha)=u(0)\right\}
$$


Proof: The validity of the first statement and (2.3) readily follows by the fact that each $|\cdot|$-convergent sequence is also $r$-convergent, and each $r$-convergent sequence either converges in $|\cdot|$ to the same limit $\alpha \neq 0$, or has two $|\cdot|$-accumulation points: 0 and $\infty$. Since the mentioned embedding is continuous and injective, it is also Borel-measurable. By Kuratowski's theorem, see Parthasarathy (1967), page 21, its inverse is also measurable and thus is the isomorphism in question. This, in particular, means that the corresponding Borel $\sigma$-fields coincide.

Definition 2.3. For a suitable $u \in C\left(\mathbb{R}_{+}, \mathcal{T}_{r}\right)$, we introduce the map $\mathbb{R}_{+} \ni \alpha \mapsto u^{\prime}(\alpha)$ meaning the usual derivative if $\alpha>0$, and the right-hand side one if $\alpha=0$. A given $u$ is said to be continuously differentiable (on $\mathbb{R}_{+}$) if $u^{\prime} \in C\left(\mathbb{R}_{+}, \mathcal{T}_{r}\right)$.

Let us consider the following functions

$$
u_{n}(\alpha)=\frac{\alpha^{2}}{1+n \alpha^{3}}, \quad \alpha \in \mathbb{R}_{+}, \quad n \in \mathbb{N} .
$$

It is clear that: (a) each $u_{n}$ is continuously differentiable, see Definition 2.3; (b) $u_{n}$ is decreasing for $\alpha>(2 / n)^{1 / 3}$. Moreover, $u_{n}(\alpha) \leq 2^{2 / 3} / 3 n^{2 / 3}$ and

$$
u_{n}^{\prime}(\alpha)=\frac{2 \alpha-n \alpha^{4}}{\left(1+n \alpha^{3}\right)^{2}}, \quad\left|u_{n}^{\prime}(\alpha)\right| \leq c / n^{1 / 3},
$$

the latter holding for some $c>0$ and all $\alpha \geq 0$.

Now let $\left\{\sigma_{k}\right\}_{k \in \mathbb{N}}=: \Sigma \subset[0,+\infty)$ be countable and such that: (i) $\sigma_{1}=0$; (ii) $\sigma_{k}<\sigma_{k+1}$ for all $k \in \mathbb{N} ; \sigma_{k} \rightarrow \bar{\sigma}<\infty$ as $k \rightarrow+\infty$. Next, for $k, n \in \mathbb{N}$, we set

$$
w_{k, n}(\alpha)=e^{-\sigma_{k} u_{n}(\alpha)} .
$$

Then $w_{k, n}$ is continuously differentiable and the following holds

$$
\left|w_{k, n}^{\prime}(\alpha)\right| \leq \frac{\bar{\sigma} c}{n^{1 / 3}} w_{k, n}(\alpha)
$$

where $c$ is the same as in (2.5).

Next, let $a$ be a finite collection of points $\alpha_{l} \in \mathbb{R}_{+}$. That is, $a=\left\{\alpha_{l}\right\}_{1 \leq l \leq m}, \alpha_{l} \leq \alpha_{l+1}$ for all $l$. For $\alpha \in a$, by $n_{a}(\alpha) \in \mathbb{N}$ we will denote the multiplicity of $\alpha$ in $a$, i.e., the number of elements of $a$ coinciding with this $\alpha$. We extend it to all $\alpha \geq 0$ by setting $n_{a}(\alpha)=0$ whenever $\alpha$ is not in $a$. Two such $a$ and $a^{\prime}$ are equal if they consist of exactly the same elements, with the same multiplicities.

Proposition 2.4. Let $a$ and $a^{\prime}$ be as just described. Then they are equal if

$$
\sum_{\alpha \in a} w_{k, n}(\alpha)=\sum_{\alpha \in a^{\prime}} w_{k, n}(\alpha)
$$

holding for all $k, n \in \mathbb{N}$.

Proof: For $a$ as above and $\zeta \in \mathbb{C}$, consider

$$
f_{n, a}(\zeta)=\sum_{\alpha \in a} \exp \left(-\zeta u_{n}(\alpha)\right), \quad n \in \mathbb{N} .
$$

Each such $f$ is an exponential type entire function. By (2.6) and (2.8) we have that $\left.\left(f_{n, a}-f_{n, a^{\prime}}\right)\right|_{\Sigma}=$ 0 , holding for all $n \in \mathbb{N}$. Since $\Sigma$ has a limiting point, this implies $f_{n, a}(\zeta)=f_{n, a^{\prime}}(\zeta)$ for all $\zeta \in \mathbb{R}$ and $n \in \mathbb{N}$. Obviously, $\lim _{\zeta \rightarrow+\infty} f_{n, a}(\zeta)=n_{a}(0)$, where $n_{a}(0) \geq 0$ is the multiplicity of $\alpha=0$ in $a$. Then the just mentioned equality yields $n_{a}(0)=n_{a^{\prime}}(0)$, and also

$$
\sum_{\alpha \in a \backslash\{0\}} \exp \left(-\zeta u_{n}(\alpha)\right)=\sum_{\alpha \in a^{\prime} \backslash\{0\}} \exp \left(-\zeta u_{n}(\alpha)\right) .
$$


Let $\alpha_{*}$ and $\alpha_{*}^{\prime}$ be the least positive elements of $a$ and $a^{\prime}$, respectively. Take $n>2 / \alpha_{b}^{3}, \alpha_{b}:=$ $\min \left\{\alpha_{*} ; \alpha_{*}^{\prime}\right\}$. Then, for such $n$ and all $\alpha>\alpha_{b}$, one has $u_{n}\left(\alpha_{b}\right)>u_{n}(\alpha)$. Now we multiply both sides of (2.9) by $e^{\zeta u_{n}\left(\alpha_{b}\right)}$ and pass to the limit $\zeta \rightarrow-\infty$. This yields that $\alpha_{*}=\alpha_{*}^{\prime}$ and $n_{a}\left(\alpha_{*}\right)=n_{a^{\prime}}\left(\alpha_{*}^{\prime}\right)$. Thereafter, we subtract the coinciding terms from both sides of (2.9) and proceed to comparing the remaining least elements of $a$ and $a^{\prime}$. This eventually yields the equality to be proved.

Let $\mathcal{A}$ be the set of all $a=\left\{\alpha_{l}\right\}_{1 \leq l \leq m}, m \in \mathbb{N}_{0}, 0 \leq \alpha_{1} \leq \alpha_{2} \leq \cdots \leq \alpha_{m}$. Define

$$
\begin{aligned}
\rho\left(a, a^{\prime}\right) & =\sum_{k, n \in \mathbb{N}} \frac{2^{-k-n} \rho_{k, n}\left(a, a^{\prime}\right)}{1+\rho_{k, n}\left(a, a^{\prime}\right)}, \\
\rho_{k, n}\left(a, a^{\prime}\right) & :=\left|\sum_{\alpha \in a} w_{k, n}(\alpha)-\sum_{\alpha \in a^{\prime}} w_{k, n}(\alpha)\right| .
\end{aligned}
$$

By Proposition 2.4 it follows that $\rho$ is a metric on $\mathcal{A}$. Each $a \in \mathcal{A}$ can be considered as a finite counting measure defined on the compact space $\left(\mathbb{R}_{+}, r\right)$, for which $a(\Delta)=\sum_{\alpha \in a} \mathbb{1}_{\Delta}(a)=|a \cap \Delta|$, holding for all Borel subsets $\Delta$. The weak topology of $\mathcal{A}$ is defined as the coarsest topology that makes continuous all the maps $a \mapsto \sum_{\alpha \in a} w(a), w \in C\left(\mathbb{R}_{+}, \mathcal{T}_{r}\right)$. In the weak topology, $\mathcal{A}$ is a closed subset of the space of all finite positive measures on $\left(\mathbb{R}_{+}, \mathcal{T}_{r}\right)$.

Proposition 2.5. $(\mathcal{A}, \rho)$ is a complete metric space. The corresponding metric topology coincides with the weak topology that turns $\mathcal{A}$ into a locally compact Polish space.

Proof: As each $w_{k, n}$ is in $C\left(\mathbb{R}_{+}, \mathcal{T}_{r}\right)$, the weak convergence of a sequence $\left\{a_{m}\right\}_{m \in \mathbb{N}} \subset \mathcal{A}$ to a certain $a \in \mathcal{A}$ yields $\rho\left(a, a_{m}\right) \rightarrow 0, m \rightarrow+\infty$. Assume now that $\left\{a_{m}\right\}_{m \in \mathbb{N}}$ is a $\rho$-Cauchy sequence. By taking $\sigma=0$ we then get from the latter that, for some $m_{*} \in \mathbb{N}$, the cardinalities of all $a_{m}$, $m>m_{*}$, coincide. By Prohorov's theorem this yields that $\left\{a_{m}\right\}_{m \in \mathbb{N}}$ contains a subsequence that weakly converges to some $a$. Hence, the whole sequence converges in $\rho$ to this $a$. Then the metric is complete and the corresponding metric topology is exactly the weak topology of $\mathcal{A}$. The separability and local compactness follow by the fact that $\left(\mathbb{R}_{+}, \mathcal{T}_{r}\right)$ is compact.

2.2. Marked configuration spaces. Let $\Gamma$ be the set of all locally finite simple configurations on $X$. That is, each $\gamma \in \Gamma$ is a subset of $X$ such that each compact $\Lambda \subset X$ contains a finite number of the elements of $\gamma$. Let now $\breve{\gamma}$ be the pair $(\gamma, n), \gamma \in \Gamma$ and $n: \gamma \rightarrow \mathbb{N}$. The value of $n$ at a given $x \in \gamma$ can be considered as the multiplicity of $x$ in $\breve{\gamma}$. That is, $\breve{\gamma}$ is a configuration with multiple locations, for which $\gamma$ is the ground configuration. Sometimes, we will write $n_{\breve{\gamma}}(x)$ to explicitly indicate that we mean the multiplicity of $x$ in the mentioned $\breve{\gamma}$. By $\breve{\Gamma}$ we denote the set of all such multiple configurations. For $\breve{\gamma}=(\gamma, n)$, we write $\gamma=p(\breve{\gamma})$. The weak-hash (vague) topology of $\breve{\Gamma}$ is defined as the coarsest topology that makes continuous all the maps $\breve{\gamma} \mapsto \sum_{x \in p(\breve{\gamma})} n(x) g(x)$, $g \in C_{\mathrm{cs}}(X)$. It is well-known, see e.g., Zessin (1983), Lemma 1.2, that with this topology $\breve{\Gamma}$ is a Polish space, whereas $\Gamma$ is a $G_{\delta}$ subset of $\breve{\Gamma}$, by which it is also Polish. Following Lenard (1973) we will also consider $\breve{\gamma}$ as configurations of point particles, in which distinct particles may have the same location. Such particles can be enumerated, which allows one to write

$$
\sum_{x \in p(\breve{\gamma})} n(x) g(x)=\sum_{x \in \breve{\gamma}} g(x)
$$

where in the second sum we mean a certain enumeration of this sort. In the same sense, we will write

$$
\sum_{x_{1} \in \breve{\gamma}} \sum_{x_{2} \in \breve{\gamma} \backslash x_{1}} \cdots \sum_{x_{m} \in \breve{\gamma} \backslash\left\{x_{1}, \ldots, x_{m-1}\right\}} g\left(x_{1}, \ldots, x_{m}\right), \quad m \in \mathbb{N},
$$

where in expressions like $\breve{\gamma} \backslash x$ we treat $x$ the singleton $\{x\}$, whereas $\breve{\gamma} \backslash x$ is the measure such that $n_{\breve{\gamma} \backslash x}(x)=n_{\breve{\gamma}}(x)-1$ and $n_{\breve{\gamma} \backslash x}(y)=n_{\breve{\gamma}}(y)$ for $y \neq x$. 
It is known, see, e.g., Zessin (1983), page 397, that there exists a collection $\left\{v_{s}\right\}_{s \in \mathbb{N}}=: \mathcal{V} \subset C_{\mathrm{cs}}^{+}(X)$ of suitable functions such that the metric

$$
d\left(\breve{\gamma}, \breve{\gamma}^{\prime}\right)=\sum_{s \in \mathbb{N}} \frac{2^{-s} d_{s}\left(\breve{\gamma}, \breve{\gamma}^{\prime}\right)}{1+d_{s}\left(\breve{\gamma}, \breve{\gamma}^{\prime}\right)}, \quad d_{s}\left(\breve{\gamma}, \breve{\gamma}^{\prime}\right):=\left|\sum_{x \in \breve{\gamma}} v_{s}(x)-\sum_{x \in \breve{\gamma}^{\prime}} v_{s}(x)\right|
$$

is complete and consistent with the weak-hash topology of $\breve{\Gamma}$. In the sequel, we will always mean this topology of $\breve{\Gamma}$. Obviously, we can and will assume that $\mathcal{V}$ contains also the following functions. Let $\delta$ be a complete metric of $X$ and $X^{\prime}$ a countable dense subset of $X$. Each $x^{\prime} \in X^{\prime}$ has a countable base of compact neighborhoods, which we denote by $D\left(x^{\prime}\right)$. Each $\Delta \in D\left(x^{\prime}\right)$ contains balls $B_{q}\left(x^{\prime}\right)=\left\{x \in X: \delta\left(x, x^{\prime}\right)<q\right\}$ with compact closures, where $q$ is a rational number satisfying $q \leq q^{\prime}$ for a $\Delta$-specific $q^{\prime} \in \mathbb{Q}$. For $x^{\prime} \in X^{\prime}, \Delta \in D\left(x^{\prime}\right), q \leq q^{\prime}$ and $\varsigma \in(0,1) \cap \mathbb{Q}$, let $v \in C_{\mathrm{cs}}^{+}(X)$ be such that: (a) $v(x) \equiv \varsigma$ for $x \in B_{q}\left(x^{\prime}\right)$; (b) $v(x)=0$ for $x \in X \backslash \Delta$. The countable set of all such functions is supposed to be a part of $\mathcal{V}$, and hence they are taken into account in (2.12). Since each $v_{s}$ has compact support, for each compact $\Lambda \subset X$ and any two configurations, $d_{s}\left(\breve{\gamma} \cap \Lambda, \breve{\gamma}^{\prime} \cap \Lambda\right)>0$ only for finitely many $s$. Here $\breve{\gamma} \cap \Lambda:=(p(\breve{\gamma}) \cap \Lambda, n)$.

For $\gamma \in \Gamma$, let $a: \gamma \rightarrow \mathcal{A}$ be a map, for which we denote

$$
|a(x)|=\sum_{\alpha \in a(x)} n_{a(x)}(\alpha) .
$$

Then the pair $\hat{\gamma}=(\gamma, a)$ is a marked configuration whose ground configuration is $\gamma$ and the mark map is $a$. By writing $\hat{x}=(x, \alpha) \in \hat{\gamma}$ we will mean that $x \in \gamma$ and $\alpha \in a(x)$. The configuration of marks $a(x)=\left\{\alpha_{1}, \ldots, \alpha_{|a(x)|}\right\}$ yields the ages of the particles located at $x \in \gamma$, whereas $|a(x)|$ is the total number of such particles. In some cases, we write $a_{\hat{\gamma}}$ to indicate that $a$ is defined on a given $\hat{\gamma}$. Let $\widehat{\Gamma}$ denote the set of all marked configurations $\hat{\gamma}$. Let also $\breve{p}: \widehat{\Gamma} \rightarrow \breve{\Gamma}$ be the map such that $\breve{p}(\gamma, a)=(\gamma,|a|)$, where $|a|(x)=|a(x)|$ see (2.13). Then $p \circ \breve{p}$ maps $\hat{\gamma}=(\gamma, a)$ into its ground configuration $\gamma$. For brevity, by writing $p(\hat{\gamma})$ we will mean $(p \circ \breve{p})(\hat{\gamma})$. Our aim now is to equip $\widehat{\Gamma}$ with a complete metric. Define

$$
\begin{aligned}
\kappa\left(\hat{\gamma}, \hat{\gamma}^{\prime}\right) & =\sum_{s, k, n \in \mathbb{N}} \frac{2^{-(s+k+n)} \kappa_{s, k, n}\left(\hat{\gamma}, \hat{\gamma}^{\prime}\right)}{1+\kappa_{s, k, n}\left(\hat{\gamma}, \hat{\gamma}^{\prime}\right)} \\
\kappa_{s, k, n}\left(\hat{\gamma}, \hat{\gamma}^{\prime}\right) & :=\left|\sum_{x \in p(\hat{\gamma})} v_{s}(x) \sum_{\alpha \in a_{\hat{\gamma}}(x)} w_{k, n}(\alpha)-\sum_{x \in p\left(\hat{\gamma}^{\prime}\right)} v_{s}(x) \sum_{\alpha \in a_{\hat{\gamma}^{\prime}}(x)} w_{k, n}(\alpha)\right| .
\end{aligned}
$$

Note that the latter can also be written as, cf. (2.11),

$$
\begin{aligned}
\kappa_{s, k, n}\left(\hat{\gamma}, \hat{\gamma}^{\prime}\right) & =\left|\sum_{\hat{x} \in \hat{\gamma}} g_{s, k, n}(\hat{x})-\sum_{\hat{x} \in \hat{\gamma}^{\prime}} g_{s, k, n}(\hat{x})\right|, \\
g_{s, k, n}(x, \alpha) & :=v_{s}(x) w_{k, n}(\alpha) .
\end{aligned}
$$

For a compact $\Lambda \subset X$, we write $\hat{\gamma} \cap \Lambda=(p(\hat{\gamma}) \cap \Lambda, a)$, where $a$ is the restriction of $a$ from $p(\hat{\gamma})$ to $p(\hat{\gamma}) \cap \Lambda$.

Proposition 2.6. For each $\varepsilon>0$, one may find a compact $\Lambda_{\varepsilon} \subset X$ such that, for any two configurations, the following holds

$$
\left|\kappa\left(\hat{\gamma}, \hat{\gamma}^{\prime}\right)-\kappa\left(\hat{\gamma} \cap \Lambda_{\varepsilon}, \hat{\gamma}^{\prime} \cap \Lambda_{\varepsilon}\right)\right|<\varepsilon .
$$

Proof: Fix $\varepsilon>0$ and then pick $s_{*} \in \mathbb{N}$ such that $2^{s_{*}}>1 / \varepsilon$. Now let $\Lambda_{\varepsilon}$ be covered by the supports of $v_{s}$ with $s \leq s_{*}$. For such $s$ and all $k, n \in \mathbb{N}$, we have $\kappa_{s, k, n}\left(\hat{\gamma}, \hat{\gamma}^{\prime}\right)=\kappa_{s, k, n}\left(\hat{\gamma} \cap \Lambda_{\varepsilon}, \hat{\gamma}^{\prime} \cap \Lambda_{\varepsilon}\right)$, see (2.14). This clearly yields (2.16). 
Since $\sigma=0$ is in $\Sigma$, by (2.6) and (2.12) we have that

$$
d\left(\breve{p}(\hat{\gamma}), \breve{p}\left(\hat{\gamma}^{\prime}\right)\right) \leq \kappa\left(\hat{\gamma}, \hat{\gamma}^{\prime}\right)
$$

Proposition 2.7. The metric space $(\widehat{\Gamma}, \kappa)$ is complete.

Proof: We begin by pointing out the following evident fact

$$
\tilde{\kappa}_{s}\left(\hat{\gamma}, \hat{\gamma}^{\prime}\right):=\sum_{k, n \in \mathbb{N}} \frac{2^{-(s+k+n)} \kappa_{s, k, n}\left(\hat{\gamma}, \hat{\gamma}^{\prime}\right)}{1+\kappa_{s, k, n}\left(\hat{\gamma}, \hat{\gamma}^{\prime}\right)} \leq \kappa\left(\hat{\gamma}, \hat{\gamma}^{\prime}\right)
$$

holding for all $s \in \mathbb{N}$ and $\hat{\gamma}, \hat{\gamma}^{\prime}$. Let now $\left\{\hat{\gamma}_{m}=\left(\gamma_{m}, a_{m}\right)\right\}_{m \in \mathbb{N}} \subset \widehat{\Gamma}$ be a $\kappa$-Cauchy sequence. By (2.17) the sequence $\left\{\breve{p}\left(\hat{\gamma}_{m}\right)\right\}_{m \in \mathbb{N}} \subset \breve{\Gamma}$ converges to some $\breve{\gamma}$. Take now $x \in p(\breve{\gamma})$ and then pick a compact $\Delta \subset X$ such that $\Delta \cap p(\breve{\gamma})=\{x\}$. For this $\Delta$, we then set $n_{m}(x)=\sum_{y \in p\left(\breve{\gamma}_{m}\right) \cap \Delta} n_{\breve{\gamma}_{m}}(y)$, $m \in \mathbb{N}$. From the convergence of $\left\{\breve{p}\left(\hat{\gamma}_{m}\right)\right\}_{m \in \mathbb{N}}$ to $\breve{\gamma}$, it follows that $n_{m}(x) \rightarrow n(x)$; hence, there exists $m_{*} \in \mathbb{N}$ such that $n_{m}(x)=n(x)$ for all $m>m_{*}$. Now we pick $x^{\prime} \in X^{\prime}$ and $q \in Q$ such that $x \in B_{q / 2}\left(x^{\prime}\right)$ and the closure of $B_{q}\left(x^{\prime}\right)$ lies in $\Delta$. Let now $v_{s} \in \mathcal{V}$ be such that $v_{s}(y)=\varsigma \in(0,1) \cap \mathbb{Q}$, $y \in B_{q / 2}\left(x^{\prime}\right)$, and $v_{s}(y)=0$ for $y \in X \backslash B_{q}\left(x^{\prime}\right)$. For these $m_{*}$ and $s,\left\{\hat{\gamma}_{m}\right\}_{m \geq m_{*}+1}$ is also a $\tilde{\kappa}_{s}$-Cauchy sequence, see (2.18), for which we have

$$
\kappa_{s, k, n}\left(\hat{\gamma}_{m}, \hat{\gamma}_{m+l}\right)=\varsigma\left|\sum_{y \in p\left(\hat{\gamma}_{m}\right) \cap \Delta} \sum_{\alpha \in \hat{a}_{m}(y)} w_{\sigma_{k}, n}(\alpha)-\sum_{y \in p\left(\hat{\gamma}_{m+l}\right) \cap \Delta} \sum_{\alpha \in \hat{a}_{m+l}(y)} w_{\sigma_{k}, n}(\alpha)\right|,
$$

holding for all $k, n \in \mathbb{N}, m>m_{*}$ and $l \in \mathbb{N}$. Here $\hat{a}_{m}:=a_{\hat{\gamma}_{m}}, \hat{a}_{m+l}:=a_{\hat{\gamma}_{m+l}}$. Let us enumerate $\hat{x}=(x, \alpha) \in \hat{\gamma}_{m} \cap \Delta$ in such a way that $\alpha_{p, m} \leq \alpha_{p+1, m}$ for all $p$. This yields $\hat{\gamma}_{m} \cap \Delta=\left\{\left(x_{1, m}, \alpha_{1, m}\right), \ldots\left(x_{n, m}, \alpha_{n, m}\right)\right\}$ with $n=n_{m}(x)=n(x)$. Similarly, we have $\hat{\gamma}_{m+l} \cap \Delta=$ $\left\{\left(x_{1, m+l}, \alpha_{1, m+l}\right), \ldots\left(x_{n, m+l}, \alpha_{n, m+l}\right)\right\}$ with the same $n$. Then $\left\{\alpha_{1, m}, \ldots \alpha_{n, m}\right\}=: a_{m} \in \mathcal{A}$, and also $\left\{\alpha_{1, m+l}, \ldots \alpha_{n, m+l}\right\}=: a_{m+l} \in \mathcal{A}$, and the latter equality can be rewritten as follows

$$
\kappa_{s, k, n}\left(\hat{\gamma}_{m}, \hat{\gamma}_{m+l}\right)=\varsigma\left|\sum_{p=1}^{n} w_{\sigma_{k}, n}\left(\alpha_{p, m}\right)-\sum_{p=1}^{n} w_{\sigma_{k}, n}\left(\alpha_{p, m+l}\right)\right|=\varsigma \rho_{k, n}\left(a_{m}, a_{m+l}\right),
$$

see (2.10). By (2.18) and (2.10) we then get

$$
\rho\left(a_{m}, a_{m+l}\right) \leq\left(2^{s} / \varsigma\right) \tilde{\kappa}_{s}\left(\hat{\gamma}_{m}, \hat{\gamma}_{m+l}\right) .
$$

By Proposition 2.5 this yields the convergence of $\left\{a_{m}\right\}_{m>m_{*}}$ to some $a(x) \in \mathcal{A}$, which holds for each $x \in \breve{\gamma}$. This defines the map $a: p(\breve{\gamma}) \rightarrow \mathcal{A}$, and hence the configuration $\hat{\gamma}=(p(\breve{\gamma}), a)$. Our aim now is to prove that $\kappa\left(\hat{\gamma}_{m}, \hat{\gamma}\right) \rightarrow 0$ as $m \rightarrow+\infty$.

Fix $\varepsilon>0$ and then pick a compact $\Lambda_{\varepsilon} \subset X$ such that (2.16) holds with $\varepsilon / 3$ in the right-hand side. Let $\Lambda_{\varepsilon}^{o}$ be its interior. Then pick compact mutually disjoint $\Delta_{x} \subset \Lambda^{o}, x \in p(\breve{\gamma}) \cap \Lambda^{o}$ such that $p(\breve{\gamma}) \cap \Delta_{x}=\{x\}$. As $\Lambda_{\varepsilon}$ is compact, $p(\breve{\gamma}) \cap \Lambda_{\varepsilon}^{o}$ is finite. Let $\left\{x_{j}\right\}_{j \leq J}$ be an enumeration of it. For brevity, we will write $\Delta_{j}$ in place of $\Delta_{x_{j}}, j=1, \ldots, J$. Similarly as above, by the convergence of $\left\{\breve{p}\left(\hat{\gamma}_{m}\right)\right\}_{m \in \mathbb{N}}$ to $\breve{\gamma}$, one finds $m_{*}$ such that $p\left(\hat{\gamma}_{m}\right) \cap \Delta_{j}$ is a singleton and $\left|\breve{p}\left(\hat{\gamma}_{m}\right) \cap \Delta_{j}\right|=: n_{m}\left(x_{j}\right)=$ $n\left(x_{j}\right)$, holding for all $m>m_{*}$ and $j \leq J$. Now we repeat the construction just made in each of $\Delta_{j}$. That is, we enumerate $\hat{\gamma}_{m} \cap \Delta_{j}=\left\{\left(x_{1, m}^{j}, \alpha_{1, m}^{j}\right), \ldots,\left(x_{n\left(x_{j}\right), m}^{j}, \alpha_{n\left(x_{j}\right), m}^{j}\right)\right\}$, and then set $a_{m}^{j}=\left\{\alpha_{1, m}^{j}, \ldots, \alpha_{n\left(x_{j}\right), m}^{j}\right\}$. Then we set $\hat{\gamma}_{*, m}=\left(\gamma_{*, m}, a_{*, m}\right)$, where $\gamma_{*, m}=p(\breve{\gamma}) \cap \Lambda_{\varepsilon}^{o}=\left\{x_{1}, \ldots, x_{J}\right\}$ and $a_{*, m}\left(x_{j}\right)=a_{m}^{j}$. In other words, the ground configuration of $\hat{\gamma}_{*, m}$ is the part of the limiting configurations $p(\hat{\gamma})$ contained in $\Lambda_{\varepsilon}^{o}$, whereas the marks are taken from the corresponding part of $\hat{\gamma}_{m}$. By the triangle inequality we then have

$$
\kappa\left(\hat{\gamma}, \hat{\gamma}_{m}\right) \leq \kappa\left(\hat{\gamma} \cap \Lambda_{\varepsilon}^{o}, \hat{\gamma}_{*, m}\right)+\kappa\left(\hat{\gamma}_{m} \cap \Lambda_{\varepsilon}^{o}, \hat{\gamma}_{*, m}\right)+\varepsilon / 3 .
$$


By (2.14), for each $s, k, n \in \mathbb{N}$, we have

$$
\begin{aligned}
\kappa_{s, k, n}\left(\hat{\gamma} \cap \Lambda_{\varepsilon}^{o}, \hat{\gamma}_{*, m}\right) & =\left|\sum_{j=1}^{J} v_{s}\left(x_{j}\right)\left[\sum_{\alpha \in a_{m}^{j}} w_{\sigma_{k}, n}(\alpha)-\sum_{\alpha \in a\left(x_{j}\right)} w_{\sigma_{k}, n}(\alpha)\right]\right| \\
& \leq J \max _{j \leq J} \rho_{k, n}\left(a_{m}^{j}, a\left(x_{j}\right)\right) .
\end{aligned}
$$

Likewise,

$$
\begin{aligned}
\kappa_{s, k, n}\left(\hat{\gamma}_{m} \cap \Lambda_{\varepsilon}^{o}, \hat{\gamma}_{*, m}\right) & =\left|\sum_{j=1}^{J}\left(\sum_{p=1}^{n\left(x_{j}\right)} v_{s}\left(x_{p, m}^{j}\right)\right) \sum_{\alpha \in a_{m}^{j}} w_{\sigma_{k}, n}(\alpha)-\sum_{j=1}^{J} v_{s}\left(x_{j}\right) \sum_{\left.\alpha \in a_{m}^{j}\right)} w_{\sigma_{k}, n}(\alpha)\right| \\
& \leq d_{s}\left(\breve{\gamma}_{m} \cap \Lambda_{\varepsilon}^{o}, \breve{\gamma} \cap \Lambda_{\varepsilon}^{o}\right) .
\end{aligned}
$$

Both latter estimates yield

$$
\begin{aligned}
\kappa\left(\hat{\gamma} \cap \Lambda_{\varepsilon}^{o}, \hat{\gamma}_{*, m}\right) & \leq J \max _{j \leq J} \rho\left(a_{m}^{j}, a\left(x_{j}\right)\right) \\
\kappa\left(\hat{\gamma}_{m} \cap \Lambda_{\varepsilon}^{o}, \hat{\gamma}_{*, m}\right) & \leq d\left(\breve{\gamma}_{m}, \breve{\gamma}\right) .
\end{aligned}
$$

By the aforementioned convergence $\breve{\gamma}_{m} \rightarrow \breve{\gamma}$ and $a_{m}^{j} \rightarrow a\left(x_{j}\right)$, one can find $m_{\varepsilon}>m_{*}$ such that the first two summands in (2.19) are smaller than $\varepsilon$ for $m>m_{\varepsilon}$, which completes the proof.

2.3. Measures and functions on configuration spaces. For $v_{s}$ and $w_{k, n}$ as in (2.14) we set

$$
\theta_{s, k, n}(x, \alpha)=\exp \left(-v_{s}(x) w_{k, n}(\alpha)\right)-1=\exp \left(-g_{s, k, n}(x, \alpha)\right)-1,
$$

see (2.15). Then $\theta_{s, k, n}(x, \alpha) \in C_{\mathrm{CS}}(\widehat{X})$ and $\theta_{s, k, n}(x, \alpha) \in(-1,0]$. Let $\Theta$ be the subset of $C_{\mathrm{cS}}(\widehat{X})$ consisting of

$$
\theta(\hat{x})=e^{-g(\hat{x})}-1, \quad g(x, \alpha)=\sum_{j} v_{s_{j}}(x) w_{k_{j}, n_{j}}(\alpha),
$$

where the latter sum runs over a finite subset of $\mathbb{N}^{3}$. That is, each such $g$ is a finite sum of $g_{s, k, n}$ defined in (2.15). Note that $\Theta$ is countable and closed under the map $\left(\theta, \theta^{\prime}\right) \mapsto\left(\theta * \theta^{\prime}\right)$, where

$$
\left(\theta * \theta^{\prime}\right)(\hat{x})=\theta(\hat{x})+\theta^{\prime}(\hat{x})+\theta(\hat{x}) \theta^{\prime}(\hat{x})=-1+(1+\theta(x))\left(1+\theta^{\prime}(x)\right) .
$$

Moreover, by (2.4), (2.6) and (2.20) it follows that

$$
g(x, \alpha) \leq g(x, 0)
$$

holding for all $\alpha \geq 0$ and $x \in X$. Now for $\theta \in \Theta$, we set

$$
F^{\theta}(\hat{\gamma})=\prod_{\hat{x} \in \hat{\gamma}}(1+\theta(\hat{x}))=\exp \left(-\sum_{\hat{x} \in \hat{\gamma}} g(\hat{x})\right), \quad \hat{\gamma} \in \widehat{\Gamma} .
$$

Then $F^{\theta}(\hat{\gamma}) \in(0,1]$ for all $\hat{\gamma} \in \widehat{\Gamma}$, and hence $F^{\theta} \in C_{\mathrm{b}}(\widehat{\Gamma})$. The set of all such functions will be denoted by $\mathcal{F}_{\Theta}$. For $\mu \in \mathcal{P}(\widehat{\Gamma})$, we then have

$$
\mu\left(F^{\theta}\right)=\int_{\widehat{\Gamma}} F^{\theta}(\hat{\gamma}) \mu(d \hat{\gamma}) \leq 1
$$

The Poisson measure $\pi_{\varrho}$ with intensity measure $\varrho$ satisfies

$$
\pi_{\varrho}\left(F^{\theta}\right)=\exp [\varrho(\theta)]=\exp \left(\int_{\widehat{X}} \theta(\hat{x}) \varrho(d \hat{x})\right) .
$$


For $\mu_{1}, \mu_{2} \in \mathcal{P}(\widehat{\Gamma})$, their convolution is defined by the expression

$$
\left(\mu_{1} \star \mu_{2}\right)(F)=\int_{\widehat{\Gamma}^{2}} F\left(\hat{\gamma}_{1} \cup \hat{\gamma}_{2}\right) \mu_{1}\left(d \hat{\gamma}_{1}\right) \mu_{2}\left(d \hat{\gamma}_{2}\right),
$$

that ought to hold for all $F \in B_{\mathrm{b}}(\widehat{\Gamma})$. For $F^{\theta}$ as in $(2.23)$, it takes the form

$$
\left(\mu_{1} \star \mu_{2}\right)\left(F^{\theta}\right)=\mu_{1}\left(F^{\theta}\right) \mu_{2}\left(F^{\theta}\right) .
$$

Recall that a set $\mathcal{F} \subset C_{\mathrm{b}}(\widehat{\Gamma})$ is called convergence determining if $\mu_{n}(F) \rightarrow \mu(F), n \rightarrow+\infty$, implies $\mu_{n} \Rightarrow \mu$, holding for each $\left\{\mu_{n}\right\}_{n \in \mathbb{N}} \subset \mathcal{P}(\widehat{\Gamma})$. It is known, see Ethier and Kurtz (1986), Theorem 4.5 , page 113 , that such $\mathcal{F}$ enjoys this property if it is closed under pointwise multiplication and is strongly separating. The latter means that, for each $\hat{\gamma} \in \widehat{\Gamma}$ and $\epsilon>0$, there exists a finite family $\left\{F_{j}\right\} \subset \mathcal{F}$ such that

$$
\inf _{\hat{\gamma}^{\prime} \in \widehat{C}_{\epsilon}} \max _{j}\left|F_{j}(\hat{\gamma})-F_{j}\left(\hat{\gamma}^{\prime}\right)\right|>0, \quad \widehat{C}_{\epsilon}:=\widehat{\Gamma} \backslash \widehat{B}_{\epsilon}(\hat{\gamma}) .
$$

Here $\widehat{B}_{\epsilon}(\hat{\gamma})=\left\{\hat{\gamma}^{\prime}: \kappa\left(\hat{\gamma}, \hat{\gamma}^{\prime}\right)<\epsilon\right\}$, see (2.14). Note that taking $\epsilon \geq 1$ does not make sense as $\kappa\left(\hat{\gamma}, \hat{\gamma}^{\prime}\right)<1$ for all configurations.

Proposition 2.8. The set $\mathcal{F}_{\Theta}$ is strongly separating and thus convergence determining.

Proof: By the very definition of $\Theta$, cf. (2.21), $\mathcal{F}_{\Theta}$ is closed under pointwise multiplication. To prove (2.27), we note that, see (2.23) and (2.15),

$$
\left|F^{\theta_{s, k, n}}(\hat{\gamma})-F^{\theta_{s, k, n}}\left(\hat{\gamma}^{\prime}\right)\right| \geq \min \left\{F^{\theta_{s, k, n}}(\hat{\gamma}) ; F^{\theta_{s, k, n}}\left(\hat{\gamma}^{\prime}\right)\right\} \kappa_{s, k, n}\left(\hat{\gamma} ; \hat{\gamma}^{\prime}\right)
$$

holding for all $\hat{\gamma}, \hat{\gamma}^{\prime} \in \widehat{\Gamma}$. Now we fix $\hat{\gamma}$ and $\epsilon \in(0,1)$ and then take $m$ such that $2^{-m}<\epsilon / 2$. For this $m$ and any $\hat{\gamma}^{\prime} \in \widehat{C}_{\epsilon}$, by (2.14) we readily conclude that

$$
\max _{(s, k, n): s+k+n \leq m} \kappa_{s, k, n}\left(\hat{\gamma}, \hat{\gamma}^{\prime}\right)>0,
$$

which by (2.28) yields the proof.

The important properties of the family $\mathcal{F}_{\Theta}$ are summarized in the following statement.

Proposition 2.9. The following is true:

(i) $\mathcal{B}(\widehat{\Gamma})=\sigma\left\{\mathcal{F}_{\Theta}\right\}$;

(ii) $B_{\mathrm{b}}(\widehat{\Gamma})$ is the bp-closure of the linear span of $\mathcal{F}_{\Theta}$;

(iii) $\mathcal{F}_{\Theta}$ is separating;

(iv) $\mathcal{F}_{\Theta}$ is convergence determining.

The proof of (i) and (ii) is standard, see Dawson (1993), Lemma 3.2.5 and Theorem 3.2.6, page 43. The proof of (iv) was done above, whereas (iii) is a direct consequence of (iv), cf. Ethier and Kurtz (1986).

Proposition 2.10. The Kolmogorov operator introduced in (1.2) has the property $L: \mathcal{F}_{\Theta} \rightarrow C_{\mathrm{b}}(\widehat{\Gamma})$.

Proof: We consider each of the summands in (1.2) - denoted by $L_{i}, i=1,2,3$ - separately. Thus,

$$
\left(L_{3} F^{\theta}\right)(\hat{\gamma})=F^{\theta}(\hat{\gamma}) \int_{X} \theta(x, 0) \chi(d x)
$$

i.e., it is just the multiplication operator by a $\theta$-dependent constant. Next,

$$
\left(L_{2} F^{\theta}\right)(\hat{\gamma})=-\sum_{x \in \hat{\gamma}} m(\hat{x}) \theta(\hat{x}) F^{\theta}(\hat{\gamma} \backslash \hat{x})=F^{\theta}(\hat{\gamma}) \Psi_{2}(\hat{\gamma})
$$


where

$$
\Psi_{2}(\hat{\gamma})=\sum_{\hat{x} \in \hat{\gamma}} \psi_{2}(\hat{x})=\sum_{\hat{x} \in \hat{\gamma}} m(\hat{x})\left(e^{g(\hat{x})}-1\right) .
$$

Let us consider the following function

$$
\phi_{\tau}(\hat{x})=g(\hat{x})-\tau m(\hat{x})\left(e^{g(\hat{x})}-1\right), \quad \tau \geq 0 .
$$

Since each $g_{s, k, n}(\hat{x})<1$, see $(2.15)$, it follows that $g(\hat{x}) \leq J_{\theta}$ where $J_{\theta}$ is just the number of summands in the sum in (2.20). At the same time, $m(\hat{x}) \leq m_{*}<\infty$. Taking this into account, we set

$$
\tau_{*}=1 / m_{*} e^{J_{\theta}}
$$

Then

$$
\phi_{\tau_{*}}(\hat{x})>0, \quad \hat{x} \in \widehat{X}
$$

Now by the simple inequality $\beta e^{-\tau \beta} \leq 1 / e \tau, \tau, \beta>0$, we have, see (2.30) and (2.31),

$$
\left(L_{2} F^{\theta}\right)(\hat{\gamma}) \leq \tau_{*}^{-1} \exp \left(-1-\sum_{\hat{x} \in \hat{\gamma}} \phi_{\tau_{*}}(\hat{x})\right) \leq m_{*} e^{J_{\theta}-1},
$$

which yields the boundedness in question. The continuity of $L_{2} F^{\theta}$ follows by the continuity of $\Psi_{2}$, which in turn follows by the fact that $\psi_{2} \in C_{\mathrm{cs}}(\widehat{X})$. Finally,

$$
\left(L_{1} F^{\theta}\right)(\hat{\gamma})=\left(-\sum_{\hat{x} \in \hat{\gamma}} g^{\prime}(\hat{x})\right) F^{\theta}(\hat{\gamma})=: \Psi_{1}(\hat{\gamma}) F^{\theta}(\hat{\gamma})
$$

By (2.20) we have

$$
g^{\prime}(x, \alpha)=\sum_{j} v_{s_{j}}(x) w_{k_{j}, n_{j}}^{\prime}(\alpha)
$$

which yields the continuity of $\Psi_{1}$. At the same time, by (2.7) we have

$$
\left|g^{\prime}(x, \alpha)\right| \leq \sum_{j} v_{s_{j}}(x)\left|w_{k_{j}, n_{j}}^{\prime}(\alpha)\right| \leq \bar{\sigma} c g(x, \alpha),
$$

which yields

$$
\left|g^{\prime}(x, \alpha)\right| \leq \bar{\sigma} c J_{\theta}
$$

and also

$$
\left|\left(L_{1} F^{\theta}\right)(\hat{\gamma})\right| \leq \bar{\sigma} c / e
$$

This completes the proof.

We summarize the estimates obtained above in the following

$$
\sup _{\hat{\gamma} \in \widehat{\Gamma}}\left|\left(L F^{\theta}\right)(\hat{\gamma})\right| \leq \chi(|\theta(\cdot, 0)|)+m_{*} e^{J_{\theta}-1}+\bar{\sigma} c / e .
$$

Note that

$$
\begin{aligned}
\chi(|\theta(x, \alpha)|):=\int_{X}|\theta(x, \alpha)| \chi(d x) & =\int_{X}\left(1-e^{-g(x, \alpha)}\right) \chi(d x) \\
& \leq \int_{X} g(x, 0) \chi(d x),
\end{aligned}
$$

see (2.22). 


\section{The Kolmogorov Equation}

3.1. Notations and useful estimates. For $\theta \in \Theta$, see (2.20), we set

$$
\begin{aligned}
& \theta_{t}(x, \alpha)=\theta(x, \alpha+t) \exp (M(x, \alpha)-M(x, \alpha+t)), \\
& M(x, \alpha)=\int_{0}^{\alpha} m(x, \beta) d \beta .
\end{aligned}
$$

Then $\widehat{X} \ni \hat{x} \mapsto \theta_{t}(\hat{x})$ is continuous and compactly supported for all $t \geq 0$. Moreover, both maps $t \mapsto \theta_{t}(\hat{x})$ and $\alpha \mapsto \theta_{t}(x, \alpha)$ are continuously differentiable and the following holds

$$
\frac{\partial}{\partial t} \theta_{t}(x, \alpha)=\frac{\partial}{\partial \alpha} \theta_{t}(x, \alpha)-m(x, \alpha) \theta_{t}(x, \alpha) .
$$

Note that

Next, we define, cf. (2.20),

$$
\left(\theta_{t}\right)_{s}(\hat{x})=\theta_{t+s}(\hat{x})
$$

$$
g_{t}(\hat{x})=-\log \left(1+\theta_{t}(\hat{x})\right)
$$

By (3.1) it follows that

$$
\left|\theta_{t}(x, \alpha)\right| \leq|\theta(x, \alpha+t)|=1-e^{-g(x, \alpha+t)} \leq 1-e^{-J_{\theta}},
$$

where $J_{\theta}$ is the same as in (2.32). By (3.4) this yields

$$
g_{t}(\hat{x}) \leq J_{\theta}, \quad t \geq 0, \quad \hat{x} \in \widehat{X} .
$$

By (3.1) we also have

$$
\left|\theta_{t}^{\prime}(x, \alpha)\right| \leq 2 m_{*}+\left|\theta^{\prime}(x, \alpha+t)\right| \leq 2 m_{*}+J_{\theta},
$$

where $m_{*}$ is as in (2.32) and the estimate $\left|\theta^{\prime}(x, \alpha)\right| \leq\left|g^{\prime}(x, \alpha)\right| \leq J_{\theta}$ was used, see (2.20) and (2.35).

Now we define

$$
F_{t}^{\theta}(\hat{\gamma})=\exp \left[\int_{0}^{t}\left(\int_{X} \theta(x, \alpha) e^{-M(x, \alpha)} \chi(d x)\right) d \alpha\right] F^{\theta_{t}}(\hat{\gamma}),
$$

with $\theta_{t}$ as in (3.1). Clearly, $F_{t}^{\theta} \in C_{\mathrm{b}}(\widehat{\Gamma})$ for all $t>0$ and $\theta \in \Theta$, and

$$
0<F_{t}^{\theta}(\hat{\gamma}) \leq 1, \quad \hat{\gamma} \in \widehat{\Gamma} .
$$

Furthermore, for all $t, s \geq 0$, the following holds, see (3.3),

$$
F_{t+s}^{\theta}=\exp \left(\int_{0}^{s} \int_{X} \theta_{u}(x, 0) \chi(d x) d u\right) F_{t}^{\theta_{s}} .
$$

Let us prove that $L F_{t}^{\theta} \in C_{\mathrm{b}}(\widehat{\Gamma})$ for all $\theta \in \Theta$ and $t \geq 0$. As in the proof of Proposition 2.10 we divide $L$ into three parts. Similarly as in (2.29) we have

$$
\left|\left(L_{3} F_{t}^{\theta}\right)(\hat{\gamma})\right| \leq \chi(|\theta(\cdot, t)|) \leq \chi(g(\cdot, 0)),
$$

see (2.37) and (3.7). Since $g_{t}(\hat{x})$ satisfies (3.5) for all $t \geq 0$, it follows that

$$
\left|\left(L_{2} F_{t}^{\theta}\right)(\hat{\gamma})\right| \leq m_{*} e^{J_{\theta}-1}
$$

holding for all $t>0$, see (2.33). The estimate of $\left|\left(L_{1} F_{t}^{\theta}\right)(\hat{\gamma})\right|$ is obtained as follows. Denote

$$
q_{t}(x, \alpha)=\exp \left(-\int_{\alpha}^{\alpha+t} m(x, \beta) d \beta\right) .
$$


Then by (3.1) we have

$$
\Phi\left(g_{t}(x, \alpha)\right)=q_{t}(x, \alpha) \Phi(g(x, \alpha+t)), \quad \Phi(b):=1-e^{-b}, \quad b \geq 0,
$$

by which we get that $g_{t}(x, \alpha) \leq g(x, \alpha+t)$. Let us prove that

$$
g_{t}(x, \alpha) e^{-g_{t}(x, \alpha)} \geq q_{t}(x, \alpha) g(x, \alpha+t) e^{-g(x, \alpha+t)} .
$$

By (3.9) this is equivalent to the fact that the function $b \mapsto b /\left(e^{b}-1\right)$ is decreasing, which is obviously the case. Now we take the $\alpha$-derivative from both sides of (3.9) and obtain

$$
\begin{aligned}
g_{t}^{\prime}(x, \alpha)=q_{t}(x, \alpha) g^{\prime}(x, \alpha+t) \exp \left(g_{t}(x, \alpha)-g(x, \alpha+t)\right) \\
+m(x, \alpha+t)\left(e^{g_{t}(x, \alpha)}-1\right)-m(x, \alpha)\left(e^{g_{t}(x, \alpha)}-1\right),
\end{aligned}
$$

that can be estimated as follows

$$
\begin{aligned}
\left|g_{t}^{\prime}(x, \alpha)\right| & =\bar{\sigma} c q_{t}(x, \alpha) g(x, \alpha+t) \exp \left(g_{t}(x, \alpha)-g(x, \alpha+t)\right)+2 m_{*}\left(e^{g_{t}(x, \alpha)}-1\right) \\
& \leq g_{t}(x, \alpha) e^{J_{\theta}}\left(\bar{\sigma} c+2 m_{*}\right)
\end{aligned}
$$

where we used (2.34), (3.5) and (3.10). Now we proceed as in obtaining (2.36), which eventually yields

$$
\left|\left(L F_{t}^{\theta}\right)(\hat{\gamma})\right| \leq \chi(g(\cdot, 0))+m_{*} e^{J_{\theta}-1}+\left(\bar{\sigma} c+2 m_{*}\right) e^{J_{\theta}}=: \ell_{\theta} .
$$

The key property of the latter estimate is that it is uniform in $t$. However, it does depend on $\theta$.

Along with the estimates derived above, we will use also the following. For $\theta \in \Theta$, the corresponding $g$ has the form as in (2.20). By (2.5) and (2.6) we have that

$$
e^{-\bar{\sigma} 2^{2 / 3} / 3} w_{k, n}(0) \leq w_{k, n}(\alpha) \leq w_{k, n}(0)
$$

which means that, cf. (2.22),

$$
\bar{c} g(x, 0) \leq g(x, \alpha) \leq g(x, 0), \quad \bar{c}:=e^{-\bar{\sigma} 2^{2 / 3} / 3} .
$$

For $\Phi$ as in (3.9), we have $b \geq \Phi(b) \geq b-b^{2} / 2, b \geq 0$, which we use together with (3.13) to obtain the following

$$
\begin{aligned}
g_{t}(\hat{x}) & \geq q_{t}(\hat{x}) \Phi(\bar{c} g(x, 0)) \geq e^{-m_{*} t} \Phi\left(\bar{c} g(x, 0) / J_{\theta}\right) \\
& \geq e^{-m_{*} t} \frac{\bar{c} g(x, 0)}{J_{\theta}}\left(1-\bar{c} g(x, 0) / 2 J_{\theta}\right) \\
& \geq e^{-m_{*} t} \bar{c}_{\theta} g(x, 0), \quad \bar{c}_{\theta}:=\bar{c} / 2 J_{\theta},
\end{aligned}
$$

where we have used the fact that $g(x, 0) \leq J_{\theta}$, see $(3.5)$.

3.2. The operator. We fix $\hat{\gamma} \in \widehat{\Gamma}$ and calculate the $t$-derivative of (3.2). This yields

$$
\begin{aligned}
\frac{\partial}{\partial t} F_{t}^{\theta}(\hat{\gamma}) & =\left(\int_{X} \theta(x, t) e^{-M(x, t)} \chi(d x)\right) F_{t}^{\theta}(\hat{\gamma})+\sum_{\hat{x} \in \hat{\gamma}} \frac{\partial \theta(\hat{x})}{\partial t} F_{t}^{\theta}(\hat{\gamma} \backslash \hat{x}) \\
& =\left(\int_{X} \theta_{t}(x, 0) \chi(d x)\right) F_{t}^{\theta}(\hat{\gamma})+\sum_{\hat{x} \in \hat{\gamma}} \frac{\partial}{\partial \alpha_{x}} F_{t}^{\theta}(\hat{\gamma})+\sum_{\hat{x} \in \hat{\gamma}} m(\hat{x})\left[F_{t}^{\theta}(\hat{\gamma} \backslash \hat{x})-F_{t}^{\theta}(\hat{\gamma})\right] \\
& =\left(L F_{t}^{\theta}\right)(\hat{\gamma}) .
\end{aligned}
$$


This means that we have found a solution of the Kolmogorov equation for (1.2) in the following sense. It is a map $t \mapsto F_{t} \in C_{\mathrm{b}}(\widehat{\Gamma})$, which is pointwise in $\hat{\gamma}$ continuously $t$-differentiable and such that the equality in (1.1) holds. Our aim now is to solve (1.1) in a suitable Banach space. Recall that the paths $t \mapsto \theta_{t}$ have the flow property (3.3), see also (3.8).

Below, by saying of a property of $\theta_{s}, s \geq 0$, holding for all $\theta$, we attribute this property to all $\theta_{s}$ given in (3.1) with $\theta$ taken from $\Theta$.

Proposition 3.1. For each $\theta \in \Theta$ and $s \geq 0$, it follows that $F_{t}^{\theta_{s}} \rightarrow F^{\theta_{s}}$ as $t \rightarrow 0$ in the norm of $C_{\mathrm{b}}(\widehat{\Gamma})$.

Proof: For each $\hat{\gamma}, s \geq 0$ and $\theta \in \Theta$, by (3.15) it follows that

$$
\begin{aligned}
F_{t}^{\theta_{s}}(\hat{\gamma})-F^{\theta_{s}}(\hat{\gamma}) & =\exp \left(-\int_{0}^{s} \int_{X} \theta_{u}(x, 0) \chi(d x) d u\right)\left[F_{t+s}^{\theta}(\hat{\gamma})-F_{s}^{\theta}(\hat{\gamma})\right] \\
& =\exp \left(-\int_{0}^{s} \int_{X} \theta_{u}(x, 0) \chi(d x) d u\right) \int_{s}^{t+s}\left(L F_{u}^{\theta}\right)(\hat{\gamma}) d u
\end{aligned}
$$

which by (3.12) yields

$$
\sup _{\hat{\gamma} \in \widehat{\Gamma}}\left|F_{t}^{\theta_{s}}(\hat{\gamma})-F^{\theta_{s}}(\hat{\gamma})\right| \leq t \ell_{\theta} \exp \left(-\int_{0}^{s} \int_{X} \theta_{u}(x, 0) \chi(d x) d u\right) .
$$

This completes the proof.

The next statement is a refinement of the one just proved.

Proposition 3.2. For each $\theta \in \Theta$ and $s \geq 0$, it follows that $L F_{t}^{\theta_{s}} \rightarrow L F^{\theta_{s}}$ as $t \rightarrow 0$ in the norm of $C_{\mathrm{b}}(\widehat{\Gamma})$.

Proof: First of all we note that the equality in the first line of (3.16) allows one to obtain the property in question by showing that $L F_{t+s}^{\theta} \rightarrow L F_{s}^{\theta}$ in the same sense.

As in the proof of Proposition 2.10, we split $L$ into three parts and consider each of them separately. Fix $\theta \in \Theta$ and then denote

$$
\dot{\eta}(u)=\int_{X} \theta_{u}(x, 0) \chi(d x), \quad \eta(t)=\int_{0}^{t} \dot{\eta}(u) d u .
$$

Then

$$
\begin{aligned}
\left|\left(L_{3} F_{t+s}^{\theta}\right)(\hat{\gamma})-\left(L_{3} F_{s}^{\theta}\right)(\hat{\gamma})\right| & \leq\left|\dot{\eta}(t+s) e^{\eta(t+s)}-\dot{\eta}(s) e^{\eta(s)}\right|+|\dot{\eta}(s)| e^{\eta(s)}\left|F^{\theta_{t+s}}(\hat{\gamma})-F^{\theta_{s}}(\hat{\gamma})\right| \\
& =: I_{1}(t)+I_{2}(t)
\end{aligned}
$$

see (3.7). Then $I_{1}(t) \rightarrow 0$ as $t \rightarrow 0$ since $\dot{\eta}(t) e^{\eta(t)}$ is a continuous function of $t$. At the same time, $I_{2}(t)$ can be estimated as in (3.17). This yields the proof for $L_{3}$.

Now we proceed to $L_{2}$, for which it follows that

$$
\begin{aligned}
\left(L_{2} F_{t}^{\theta_{s}}\right)(\hat{\gamma})-\left(L_{2} F^{\theta_{s}}\right)(\hat{\gamma}) & =J_{t}(\hat{\gamma})+K_{t}(\hat{\gamma}), \\
J_{t}(\hat{\gamma}) & =\sum_{\hat{x} \in \hat{\gamma}} \frac{m(\hat{x})\left(-\theta_{t+s}(\hat{x})\right)}{1+\theta_{t+s}(\hat{x})}\left[F_{t}^{\theta_{s}}(\hat{\gamma})-F^{\theta_{s}}(\hat{\gamma})\right] \\
K_{t}(\hat{\gamma}) & =F^{\theta_{s}}(\hat{\gamma}) \sum_{\hat{x} \in \hat{\gamma}} \frac{m(\hat{x})\left(\theta_{s}(\hat{x})-\theta_{t+s}(\hat{x})\right)}{\left(1+\theta_{t+s}(\hat{x})\right)\left(1+\theta_{s}(\hat{x})\right)} .
\end{aligned}
$$


By (3.6) and (3.18) we have

$$
\begin{aligned}
F_{t}^{\theta_{s}}(\hat{\gamma})-F^{\theta_{s}}(\hat{\gamma}) & =\left(e^{\eta(t+s)-\eta(s)}-1\right) F^{\theta_{t+s}}(\hat{\gamma})+F^{\theta_{t+s}}(\hat{\gamma})-F^{\theta_{s}}(\hat{\gamma}) \\
& =: \Upsilon_{1}(t, \hat{\gamma})+\Upsilon_{2}(t, \hat{\gamma}) .
\end{aligned}
$$

By (2.37) and (3.1), (3.18) it follows that

$$
\left|e^{\eta(t+s)-\eta(s)}-1\right| \leq t \chi(g(\cdot, 0))
$$

which then yields

$$
\left|\Upsilon_{1}(t, \hat{\gamma})\right| \leq t \chi(g(\cdot, 0)) \exp \left(-\sum_{\hat{x} \in \hat{\gamma}} g_{t+s}(\hat{x})\right)
$$

To estimate $\Upsilon_{2}$ we write

$$
h_{t}(\hat{x})=\min \left\{g_{t+s}(\hat{x}) ; g_{s}(\hat{x})\right\} .
$$

Then by (3.11) and (3.14) we get

$$
\begin{aligned}
\left|\Upsilon_{2}(t, \hat{\gamma})\right| & \leq \exp \left(-\sum_{\hat{x} \in \hat{\gamma}} h_{t}(\hat{x})\right) \sum_{\hat{x} \in \hat{\gamma}}\left|g_{t+s}(\hat{x})-g_{s}(\hat{x})\right| \\
& \leq e^{J_{\theta}}\left(\bar{\sigma} c+2 m_{*}\right) \exp \left(-\sum_{\hat{x} \in \hat{\gamma}} h_{t}(\hat{x})\right) \sum_{\hat{x} \in \hat{\gamma}} \int_{s}^{t+s} g_{u}(x, \alpha) d u \\
& \leq e^{J_{\theta}}\left(\bar{\sigma} c+2 m_{*}\right) \exp \left(-e^{-m_{*} t} \bar{c}_{\theta} \sum_{\hat{x} \in \hat{\gamma}} g(x, 0)\right) \sum_{\hat{x} \in \hat{\gamma}} t g(x, 0) \\
& =: t C_{\theta} e^{-\bar{c}_{\theta}(t) \Psi_{0}(\hat{\gamma})} \Psi_{0}(\hat{\gamma}), \quad \bar{c}_{\theta}(t):=e^{-m_{*} t} \bar{c}_{\theta}, \quad \Psi_{0}(\hat{\gamma}):=\sum_{\hat{x} \in \hat{\gamma}} g(x, 0) .
\end{aligned}
$$

At the same time,

$$
0 \leq \sum_{\hat{x} \in \hat{\gamma}} \frac{m(\hat{x})\left(-\theta_{t+s}(\hat{x})\right.}{1+\theta_{t+s}(\hat{x})} \leq m_{*} e^{J_{\theta}} \sum_{\hat{x} \in \hat{\gamma}} g_{t+s}(\hat{x})=: m_{*} e^{J_{\theta}} \Psi_{1}(t, \hat{\gamma}) .
$$

Thereafter, we have

$$
\begin{aligned}
\left|J_{t}(\hat{\gamma})\right| & \leq t m_{*} e^{J_{\theta}} \chi(g(\cdot, 0)) \Psi_{1}(t, \hat{\gamma}) e^{-\Psi_{1}(t, \hat{\gamma})}+t C_{\theta} m_{*} e^{J_{\theta}} \Psi_{1}(t, \hat{\gamma}) \Psi_{0}(\hat{\gamma}) e^{-\bar{c}_{\theta}(t) \Psi_{0}(\hat{\gamma})} \\
& =: \Pi_{1}(t, \hat{\gamma})+\Pi_{2}(t, \hat{\gamma}),
\end{aligned}
$$

where $C_{\theta}, \bar{c}_{\theta}(t)$ and $\Psi_{0}$ are as in (3.23). Then

$$
\Pi_{1}(t, \hat{\gamma}) \leq t m_{*} e^{J_{\theta}-1} \chi(g(\cdot, 0)) \rightarrow 0, \quad t \rightarrow+\infty .
$$

Let $t_{\theta}>0$ be the (unique) solution of $\bar{c}_{\theta} e^{-m_{*} t}=2 t^{1 / 3}$. Then for $t \leq t_{\theta}$, we have

$$
\begin{aligned}
\Pi_{2}(t, \hat{\gamma}) & \leq t C_{\theta} m_{*} e^{J_{\theta}}\left[\Psi_{0}(\hat{\gamma})\right]^{2} e^{-\bar{c}_{\theta}(t) \Psi_{0}(\hat{\gamma})} \\
& \leq t^{1 / 3} C_{\theta} m_{*} e^{J_{\theta}-2} \exp \left(-\left(\bar{c}_{\theta}(t)-2 t^{1 / 3}\right) \Psi_{0}(\hat{\gamma})\right) \leq t^{1 / 3} C_{\theta} m_{*} e^{J_{\theta}-2},
\end{aligned}
$$

which yields the convergence

$$
\sup _{\hat{\gamma} \in \widehat{\Gamma}}\left|J_{t}(\hat{\gamma})\right| \rightarrow 0, \quad t \rightarrow 0
$$


Now we turn to $K_{t}(\hat{\gamma})$. First, by (3.2) we have

$$
\left|\theta_{s}(\hat{x})-\theta_{t+s}(\hat{x})\right|=\left|\int_{s}^{t+s}\left(\frac{\partial}{\partial \alpha} \theta_{u}(x, \alpha)-m(x, \alpha) \theta_{u}(x, \alpha)\right) d u\right| .
$$

Next, by (3.4) and (3.11) it follows that

$$
\begin{aligned}
\left|\frac{\partial}{\partial \alpha} \theta_{u}(x, \alpha)\right|=e^{-g_{u}(x, \alpha)}\left|g_{u}^{\prime}(x, \alpha)\right| & \leq e^{J_{\theta}}\left(\bar{\sigma} c+2 m_{*}\right) g_{u}(x, \alpha) \\
& \leq e^{J_{\theta}}\left(\bar{\sigma} c+2 m_{*}\right) g(x, 0)
\end{aligned}
$$

and also

$$
\left|m(x, \alpha) \theta_{u}(x, \alpha)\right| \leq m_{*} g_{u}(x, \alpha) \leq m_{*} g(x, 0) .
$$

The latter two estimates yield

$$
\operatorname{LHR}(3.25) \leq e^{J_{\theta}}\left(\bar{c}+3 m_{*}\right) g(x, 0) .
$$

By (3.13) this yields

$$
\begin{aligned}
K_{t}(\hat{\gamma}) & \leq t m_{*}\left(\bar{\sigma}+3 m_{*}\right) e^{3 J_{\theta}} \Psi_{0}(\hat{\gamma}) e^{-\bar{c} \Psi_{0}(\hat{\gamma})} \\
& \leq t m_{*}\left(\bar{\sigma}+3 m_{*}\right) e^{3 J_{\theta}-1} / \bar{c} \rightarrow 0, \quad t \rightarrow+\infty
\end{aligned}
$$

By (3.20) this completes the proof for $L_{2}$.

Next, we write

$$
\begin{aligned}
\left(L_{1} F_{t}^{\theta_{s}}\right)(\hat{\gamma})-\left(L_{1} F^{\theta_{s}}\right)(\hat{\gamma}) & =Q_{t}(\hat{\gamma})+R_{t}(\hat{\gamma}), \\
Q_{t}(\hat{\gamma}) & =\sum_{\hat{x} \in \hat{\gamma}} \theta_{t+s}^{\prime}(\hat{x})\left[F_{t}^{\theta_{s}}(\hat{\gamma} \backslash \hat{x})-F^{\theta_{s}}(\hat{\gamma} \backslash \hat{x})\right], \\
R_{t}(\hat{\gamma}) & =\sum_{\hat{x} \in \hat{\gamma}}\left[\theta_{t+s}^{\prime}(\hat{x})-\theta_{s}^{\prime}(\hat{x})\right] F^{\theta_{s}}(\hat{\gamma} \backslash \hat{x}) .
\end{aligned}
$$

Then

$$
\begin{aligned}
Q_{t}(\hat{\gamma}) & =-\sum_{\hat{x} \in \hat{\gamma}} g_{t+s}^{\prime}(\hat{x})\left[F_{t}^{\theta_{s}}(\hat{\gamma})-F^{\theta_{s}}(\hat{\gamma})\right]+F^{\theta_{s}}(\hat{\gamma}) \sum_{\hat{x} \in \hat{\gamma}} g_{t+s}^{\prime}(\hat{x})\left[e^{g_{s}(\hat{x})-g_{t+s}(\hat{x})}-1\right] \\
& =: Q_{t}^{(1)}(\hat{\gamma})+Q_{t}^{(2)}(\hat{\gamma}) .
\end{aligned}
$$

By (3.11) and then by (3.21), (3.22), (3.23) we get

$$
\begin{aligned}
\left|Q_{t}^{(1)}(\hat{\gamma})\right| \leq & e^{J_{\theta}}\left(\bar{\sigma} c+2 m_{*}\right)\left(\Upsilon_{1}(t, \hat{\gamma})+\Upsilon_{2}(t, \hat{\gamma})\right) \sum_{\hat{x} \in \hat{\gamma}} g_{t+s}(\hat{x}) \\
\leq & t \chi(g(\cdot, 0)) e^{J_{\theta}}\left(\bar{\sigma} c+2 m_{*}\right)\left(\sum_{\hat{x} \in \hat{\gamma}} g_{t+s}(\hat{x})\right) \exp \left(-\sum_{\hat{x} \in \hat{\gamma}} g_{t+s}(\hat{x})\right) \\
& \quad+t C_{\theta} e^{J_{\theta}}\left(\bar{\sigma} c+2 m_{*}\right) e^{-\bar{c}_{\theta}(t) \Psi_{0}(\hat{\gamma})}\left[\Psi_{0}(\hat{\gamma})\right]^{2} \\
=: & \Xi_{t}^{(1)}(\hat{\gamma})+\Xi_{t}^{(2)}(\hat{\gamma}) .
\end{aligned}
$$

Then

$$
\Xi_{t}^{(1)}(\hat{\gamma}) \leq t \chi(g(\cdot, 0)) e^{J_{\theta}-1}\left(\bar{\sigma} c+2 m_{*}\right) \rightarrow 0, \quad t \rightarrow 0
$$


and also, cf. (3.24),

$$
\begin{aligned}
\Xi_{t}^{(2)}(\hat{\gamma}) & \leq t^{1 / 3} C_{\theta} e^{J_{\theta}-2}\left(\bar{\sigma} c+2 m_{*}\right) \exp \left(-\left(\bar{c}_{\theta}(t)-2 t^{1 / 3}\right) \Psi_{0}(\hat{\gamma})\right) \\
& \leq t^{1 / 3} C_{\theta} m_{*} e^{J_{\theta}-2}\left(\bar{\sigma} c+2 m_{*}\right),
\end{aligned}
$$

for $t \leq t_{\theta}$. The latter two estimates yield

$$
\sup _{\hat{\gamma} \in \widehat{\Gamma}}\left|Q_{t}^{(1)}(\hat{\gamma})\right| \rightarrow 0, \quad t \rightarrow 0 .
$$

Next, by (3.5), (3.11) and (3.13) we have

$$
\begin{aligned}
& \left|Q_{t}^{(2)}(\hat{\gamma})\right| \leq e^{J_{\theta}} F^{\theta_{s}}(\hat{\gamma}) \sum_{\hat{x} \in \hat{\gamma}}\left|g_{t+s}^{\prime}(\hat{x})\right|\left|g_{t+s}(\hat{x})-g_{s}(\hat{x})\right| \\
& \leq e^{3 J_{\theta}}\left(\bar{\sigma} c+2 m_{*}\right)^{2} F^{\theta_{s}}(\hat{\gamma}) \sum_{\hat{x} \in \hat{\gamma}} g_{t+s}(\hat{x}) \int_{s}^{t+s} g_{u}(\hat{x}) d u \\
& \leq t J_{\theta} e^{3 J_{\theta}}\left(\bar{\sigma} c+2 m_{*}\right)^{2} \Psi_{0}(\hat{\gamma}) \exp \left(-\bar{c} \Psi_{0}(\hat{\gamma})\right) \\
& \leq t J_{\theta} e^{3 J_{\theta}}\left(\bar{\sigma} c+2 m_{*}\right)^{2} / \bar{c} \rightarrow 0, \quad t \rightarrow 0,
\end{aligned}
$$

which together with (3.27) yields

$$
\sup _{\hat{\gamma} \in \widehat{\Gamma}} \mid Q_{t}(\hat{\gamma} \mid \rightarrow 0, \quad t \rightarrow 0 .
$$

Now we turn to estimating $R_{t}$. By 3.1) we have

$$
\begin{aligned}
& \left|\theta_{t+s}^{\prime}(x, \alpha)-\theta_{s}^{\prime}(x, \alpha)\right| \\
& \leq\left|\theta^{\prime}(x, \alpha+t+s)\right| \cdot\left|q_{t+s}(x, \alpha)-q_{s}(x, \alpha)\right|+\left|\theta^{\prime}(x, \alpha+t+s)-\theta^{\prime}(x, \alpha+s)\right| \\
& \quad \quad+|m(x, \alpha+t+s)-m(x, \alpha+s)| \cdot\left|\theta_{t+s}(x, \alpha)\right|+|m(x, \alpha+s)-m(x, \alpha)| \cdot\left|\theta_{s}(x, \alpha)\right| \\
& =: \delta_{1}(t, \hat{x})+\delta_{2}(t, \hat{x})+\delta_{3}(t, \hat{x})+\delta_{4}(t, \hat{x}) .
\end{aligned}
$$

By (3.11) we have

$$
\begin{aligned}
\delta_{1}(t, \hat{x}) & \leq e^{-g(x, \alpha+t+s)}\left|g^{\prime}(x, \alpha+t+s)\right| \int_{\alpha+s}^{\alpha+t+s} m(x, \beta) d \beta \\
& \leq t m_{*} e^{J_{\theta}}\left(\bar{\sigma} c+2 m_{*}\right) g(x, 0) .
\end{aligned}
$$

To estimate $\delta_{2}$, by (3.4) we first get

$$
\theta^{\prime}(\hat{x})=-g^{\prime}(\hat{x}) e^{-g(\hat{x})},
$$

by which we then obtain

$$
\begin{aligned}
\delta_{2}(t, \hat{x}) & \leq\left|g^{\prime}(x, \alpha+t+s)-g^{\prime}(x, \alpha+s)\right| e^{-g(x, \alpha+t+s)}+\left|g^{\prime}(x, \alpha+s)\right|\left|e^{-g(x, \alpha+t+s)}-e^{-g(x, \alpha+s)}\right| \\
& \leq\left|g^{\prime}(x, \alpha+t+s)-g^{\prime}(x, \alpha+s)\right|+\left|g^{\prime}(x, \alpha+s)\right||g(x, \alpha+t+s)-g(x, \alpha+s)| \\
& =: \delta_{2,1}(t, \hat{x})+\delta_{2,2}(t, \hat{x}) .
\end{aligned}
$$


Now we recall that $g(x, \alpha)$ is as in (2.20) with $w_{k, n}$ defined in (2.6). Thus, we can write

$$
\delta_{2,1}(t, \hat{x}) \leq \sum_{j} v_{s_{j}}(x) \int_{s}^{t+s}\left|w_{k_{j}, n_{j}}^{\prime \prime}(\alpha+u)\right| d u .
$$

For each $k$ and $n$, we have

$$
\begin{aligned}
\left|w_{k, n}^{\prime \prime}(\alpha)\right| & =\left|-\sigma_{k} u_{n}^{\prime \prime}(\alpha) e^{-\sigma_{k} u_{n}(\alpha)}+\left[\sigma_{k} u_{n}^{\prime}(\alpha)\right]^{2} e^{-\sigma_{k} u_{n}(\alpha)}\right| \\
& \leq \bar{\sigma}\left|u_{n}^{\prime \prime}(\alpha)\right|+\left[\bar{\sigma} u_{n}^{\prime}(\alpha)\right]^{2} \leq \bar{C}
\end{aligned}
$$

holding for some $\bar{C}>0$ that is independent of $k, n$ and $\alpha$. The latter conclusion follows by (2.7) and the fact that $\left|u_{n}^{\prime \prime}(\alpha)\right| \leq 2 \phi\left(n \alpha^{3}\right)$ with $\phi(\beta)=\left(1+\beta^{2}\right) /(1+\beta)^{3}, \beta \geq 0$. Then by (3.32) we get

$$
\delta_{2,1}(t, \hat{x}) \leq t \bar{C} g(x, 0) .
$$

At the same time, by (2.34) and (2.35) it follows that

$$
\delta_{2,2}(t, \hat{x}) \leq\left|g^{\prime}(x, \alpha+s)\right| \int_{s}^{t+s}\left|g^{\prime}(x, \alpha+u)\right| d u \leq t(\bar{\sigma} c)^{2} J_{\theta} g(x, 0),
$$

which together with (3.33) yields

$$
\delta_{2}(t, \hat{x}) \leq t\left[\bar{C}+(\bar{\sigma} c)^{2} J_{\theta}\right] g(x, 0) .
$$

Finally, (1.3) and (3.4), (3.13) we have

$$
\delta_{3}(t, \hat{x}) \leq \varkappa(t) g_{t}(\hat{x}) \leq \varkappa(t) g(x, 0) .
$$

The same estimate holds true also for $\delta_{4}(t, \hat{x})$. Then by (3.30) and (3.31), (3.34) we have that

$$
\left|\theta_{t}^{\prime}(x, \alpha)-\theta^{\prime}(x, \alpha)\right| \leq \omega(t) g(x, 0), \quad \omega(t) \rightarrow 0, \quad t \rightarrow 0 .
$$

holding for some continuous function $\omega$ and all $\hat{x} \in \widehat{X}$. Now we use this in (3.26) and obtain, cf. (3.28)

$$
\left|R_{t}(\hat{\gamma})\right| \leq \omega(t) e^{J_{\theta}} F^{\theta}(\hat{\gamma}) \Psi_{0}(\hat{\gamma}) \leq \omega(t) e^{J_{\theta}} \Psi_{0}(\hat{\gamma}) e^{-\bar{c} \Psi_{0}(\hat{\gamma})} \leq \omega(t) e^{J_{\theta}} / \bar{c},
$$

which together with (3.29) yields

$$
\sup _{\hat{\gamma} \in \widehat{\Gamma}}\left|\left(L_{1} F_{t}^{\theta}\right)(\hat{\gamma})-\left(L_{1} F^{\theta}\right)(\hat{\gamma})\right| \rightarrow 0, \quad t \rightarrow 0 .
$$

This completes the whole proof.

3.3. The domain. We recall that $\Theta$ consists of the functions as in (2.20) and the countable collection $\mathcal{F}_{\Theta} \subset C_{\mathrm{b}}(\widehat{\Gamma})$ consists of the functions introduced in (2.23). It has a number of useful properties established in Propositions 2.8 and 2.9. Let $\mathcal{C}_{0}$ be the linear span of the set $\left\{F^{\theta_{s}}: s \in \mathbb{Q}_{+}, \theta \in \Theta\right\}$, i.e., each $F \in \mathcal{C}_{0}$ is a finite linear combination of $F^{\theta_{s}}$, with positive rational $s$ and $\theta_{s}$ given in (3.1) with all possible choices of $\theta \in \Theta$.

Remark 3.3. The set $\mathcal{C}_{0}$ is countable. It enjoys all the properties mentioned in Proposition 2.9.

Now we set

$$
\mathcal{C}=\overline{\mathcal{C}_{0}},
$$

i.e., $\mathcal{C}$ is the closure of $\mathcal{C}_{0}$ in the norm of $C_{\mathrm{b}}(\widehat{\Gamma})$, which we denote $\|\cdot\|$. With this norm it is then a separable Banach space. 
For $\lambda>0$ and $\theta \in \Theta$ and $s \geq 0$, we define, cf. (3.6),

$$
\begin{aligned}
F_{\lambda, \theta_{s}}(\hat{\gamma}) & =\int_{0}^{+\infty} e^{-\lambda t} F_{t}^{\theta_{s}}(\hat{\gamma}) d t \\
& =\int_{0}^{+\infty} \exp \left[-\lambda t+\int_{0}^{t}\left(\int_{X} \theta_{s}(x, \alpha) e^{-M(x, \alpha)} \chi(d x)\right) d \alpha\right] F^{\theta_{t+s}}(\hat{\gamma}) d t
\end{aligned}
$$

Since $\theta_{s}(x, \alpha) \leq 0$ and $F_{t}^{\theta_{s}}$ satisfies (3.7), the above integral converges for each $\hat{\gamma}$. By the dominated convergence theorem and the boundedness $F_{t}^{\theta_{s}}(\hat{\gamma}) \leq 1$ it follows that $F_{\lambda, \theta_{s}} \in C_{\mathrm{b}}(\widehat{\Gamma})$. Moreover, it can also be understood as the Bochner integral in the latter Banach space. Therefore, $F_{\lambda, \theta}$ can be approximated in $\|\cdot\|$ by the Riemann integral sums centered at rational $t$, which means that

$$
F_{\lambda, \theta_{s}} \in \mathcal{C}, \quad \text { for all } s \geq 0 \text { and } \lambda>0 .
$$

At the same time, we also have

$$
0<F_{\lambda, \theta_{s}}(\hat{\gamma})<1 / \lambda, \quad \hat{\gamma} \in \widehat{\Gamma} .
$$

By the dominated convergence theorem one readily proves that the map $(0,+\infty) \ni \alpha \mapsto F_{\lambda, \theta_{s}}(\hat{\gamma} \backslash$ $\hat{x} \cup(x, \alpha)) \in \mathbb{R}$ is continuously differentiable for each $\hat{\gamma}$ and $\hat{x} \in \hat{\gamma}$.

Lemma 3.4. For each $\lambda>0, s \geq 0$ and $\theta \in \Theta$, the following holds

$$
L F_{\lambda, \theta_{s}}=\lambda F_{\lambda, \theta_{s}}-F^{\theta_{s}} \text {. }
$$

Proof: By (3.6) and (3.37) we have

$$
\begin{aligned}
L F_{\lambda, \theta_{s}} & =L \int_{0}^{+\infty} e^{-\lambda t} F_{t}^{\theta_{s}} d t=\int_{0}^{+\infty} e^{-\lambda t} L F_{t}^{\theta_{s}} d t \\
& =\int_{0}^{+\infty} e^{-\lambda t} \frac{\partial}{\partial t} F_{t}^{\theta_{s}} d t=-F^{\theta_{s}}+\lambda F_{\lambda, \theta_{s}},
\end{aligned}
$$

where we have taken into account the upper bound in (3.39). The commutation $L \int=\int L$ can be justified by means of the Lebesgue dominated convergence theorem.

Lemma 3.5. For each $\theta \in \Theta$ and $s \geq 0$, it follows that $\left\|\lambda F_{\lambda, \theta_{s}}-F^{\theta_{s}}\right\| \rightarrow 0$ and $\left\|\lambda L F_{\lambda, \theta_{s}}-L F^{\theta_{s}}\right\| \rightarrow$ 0 as $\lambda \rightarrow+\infty$.

Proof: In view of (3.39), $\left\{\lambda F_{\lambda, \theta_{s}}: \lambda>0\right\}$ is bounded. By (3.37) we have

$$
\lambda F_{\lambda, \theta_{s}}(\hat{\gamma})=\int_{0}^{+\infty} \exp \left(-t+\int_{0}^{\varepsilon t}\left(\int_{X} \theta_{\alpha+s}(x, 0) \chi(d x)\right) d \alpha\right) F^{\theta_{\varepsilon t+s}}(\hat{\gamma}) d t, \quad \varepsilon:=\lambda^{-1} .
$$

Then by (3.17) it follows that

$$
\left|\lambda F_{\lambda, \theta_{s}}(\hat{\gamma})-F^{\theta_{s}}(\hat{\gamma})\right| \leq \int_{0}^{+\infty} e^{-t}\left|F_{\varepsilon t}^{\theta_{s}}(\hat{\gamma})-F^{\theta_{s}}(\hat{\gamma})\right| d t \leq \ell_{\theta} / \lambda
$$

which yields that $\left\|\lambda F_{\lambda, \theta_{s}}-F^{\theta_{s}}\right\| \rightarrow 0$ as $\lambda \rightarrow+\infty$. In the same way, by Proposition 3.2 we have, cf. (3.35), that

$$
\left\|\lambda L F_{\lambda, \theta_{s}}-L F^{\theta_{s}}\right\| \leq \tilde{\omega}(1 / \lambda) \rightarrow 0, \quad \lambda \rightarrow+\infty
$$

holding for an appropriate continuous $\tilde{\omega}$ such that $\tilde{\omega}(\epsilon) \rightarrow 0$ as $\epsilon \rightarrow 0$.

Let $\mathcal{D}_{0}(L)$ denote the linear span of the set $\left\{F_{\lambda, \theta_{s}}: \lambda>0, s \geq 0, \theta \in \Theta\right\}$. By Lemma 3.5 and (3.36) it follows that

$$
\mathcal{C}_{0} \subset \overline{\mathcal{D}_{0}(L)}
$$


i.e., $\mathcal{C}_{0}$ is contained in the closure of $\mathcal{D}_{0}(L)$ in the norm of $C_{\mathrm{b}}(\widehat{\Gamma})$. Hence, $\mathcal{D}_{0}(L)$ is a dense subset of the Banach space $\mathcal{C}$, see (3.36). Define

$$
\|F\|_{L}=\|F\|+\|L F\|, \quad F \in \mathcal{D}_{0}(L),
$$

where - as above $-\|\cdot\|$ is the norm of $C_{\mathrm{b}}(\widehat{\Gamma})$.

Definition 3.6. By the domain of the Kolmogorov operator $L$, denoted by $\mathcal{D}(L)$, we mean the closure of $\mathcal{D}_{0}(L)$ in the graph-norm introduced in (3.43).

Lemma 3.7. The operator $(L, \mathcal{D}(L))$ is closed and densely defined in $\mathcal{C}$. Its resolvent set contains $(0,+\infty)$ and $\mathcal{C}_{0} \subset \mathcal{D}(L)$.

Proof: In view of (3.38) and (3.42), the $\|\cdot\|$-closure of $\mathcal{D}(L)$ is $\mathcal{C}$. The closedness of $(L, \mathcal{D}(L))$ is immediate, and the inclusion $\mathcal{C}_{0} \subset \mathcal{D}(L)$ follows by the second part of Lemma 3.5. By (3.40) it follows that the resolvent of $L$, denoted $R_{\lambda}(L)$, has the property

$$
R_{\lambda}(L) F^{\theta_{s}}=F_{\lambda, \theta_{s}}, \quad \lambda>0, \quad s \geq 0, \quad \theta \in \Theta,
$$

by which and (3.39) we also have that the operator norm of $R_{\lambda}(L)$ satisfies $\left\|R_{\lambda}(L)\right\| \leq 1 / \lambda$ as $F^{\theta_{s}}$ form a dense subset of $\mathcal{C}$. This completes the whole proof.

3.4. Solving the Kolmogorov equation. The result obtained in Lemma 3.7 allows one to solve the Kolmogorov equation (1.1) in the following sense.

Theorem 3.8. Let $(L, \mathcal{D}(L))$ and $\mathcal{C}$ be as in Lemma 3.7. Then, for each $F \in \mathcal{D}(L)$, there exists a unique continuously differentiable map $[0,+\infty) \ni t \mapsto F_{t} \in \mathcal{D}(L) \subset \mathcal{C}$, which solves (1.1) with $F_{0}=F$. In particular, for $F=F^{\theta_{s}}$, with $F^{\theta_{s}}$ as in (3.1), $s \geq 0$ and $\theta \in \Theta$, the solution has the following explicit form, cf. (3.6)

$$
F_{t}(\hat{\gamma})=\exp \left[\int_{0}^{t}\left(\int_{X} \theta_{s}(x, \alpha) \chi(d x)\right) d \alpha\right] F^{\theta_{t+s}}(\hat{\gamma}) .
$$

Proof: By the celebrated Hille-Yosida theorem, see, e.g, Pazy (1983), page 8, $(L, \mathcal{D}(L))$ is the generator of a $C_{0}$-semigroup of bounded linear operators $S(t): \mathcal{C} \rightarrow \mathcal{C}$ such that $\|S(t)\|=1$ and the solution in question is $F_{t}=S(t) F$, the uniqueness of which is also a standard fact, see Pazy (1983), Theorem 1.3, page 102. The validity of (3.44) follows by the calculations as in (3.15).

\section{The Result}

4.1. Poisson measures and transition functions. We begin by recalling, see Proposition 2.9, that the class of functions $\mathcal{F}_{\Theta}$, see (2.23), is separating, i.e., if $\mu_{1}(F)=\mu_{2}(F)$ for all $F \in \mathcal{F}_{\Theta}$, then $\mu_{1}=\mu_{2}$, that holds for each pair $\mu_{1}, \mu_{2} \in \mathcal{P}(\widehat{\Gamma})$. Next, for $t \geq 0, \mu \in \mathcal{P}(\widehat{\Gamma})$ and $\theta \in \Theta$, we define $\mu^{t} \in \mathcal{P}(\widehat{\Gamma})$ by the relation

$$
\mu^{t}\left(F^{\theta}\right)=\mu\left(F^{\theta_{t}}\right)
$$

Recall that, for a positive Radon measure $\varrho$ on $\widehat{X}$, the Poisson measure $\pi_{\varrho}$ with intensity measure $\varrho$ is defined in (2.24). For $t \geq 0$, we then introduce the Poisson measure $\pi_{t}=\pi_{\varrho}$ by defining its intensity measure

$$
\varrho_{t}(d \hat{x})=\mathbb{1}_{[0, t)}(\alpha) \exp (-M(\hat{x})) \chi(d x) d \alpha,
$$

where $\chi$ is the same as in (1.2), $d \alpha$ is the Lebesgue measure on $\mathbb{R}_{+}$and $M$ is as in (3.1). Note that $\pi_{0}(\{\varnothing\})=1$ since $\pi_{0}\left(F^{\theta}\right)=1$ for all $\theta$. Then we set, see (2.25),

$$
\mu_{t}=\pi_{t} \star \mu^{t}, \quad t \geq 0 .
$$


Let $\delta_{\hat{\gamma}}$ be the Dirac measure centered at a given $\hat{\gamma} \in \widehat{\Gamma}$. Then

$$
p_{t}^{\hat{\gamma}}:=\pi_{t} \star \delta_{\hat{\gamma}}^{t}
$$

is a transition function, cf. Ethier and $\operatorname{Kurtz}(1986)$, page 156. Indeed, $p_{t}^{\hat{\gamma}} \in \mathcal{P}(\widehat{\Gamma}), p_{0}^{\hat{\gamma}}=\delta_{\hat{\gamma}}$ and the measurability of the map $(t, \hat{\gamma}) \mapsto p_{t}^{\hat{\gamma}}(B) \in \mathbb{R}, B \in \mathcal{B}(\widehat{\Gamma})$, follows by the measurability of $(t, \hat{\gamma}) \mapsto \delta_{\hat{\gamma}}^{t}(B) \in \mathbb{R}$ and the continuity of $t \mapsto \pi_{t}(B) \in \mathbb{R}$. In view of the separating property of $\mathcal{F}_{\Theta}$, see Proposition 2.9, item (iii), the flow property of $\left\{p_{t}^{\hat{\gamma}}\right\}_{t \geq 0}$ can be obtained by showing that

$$
p_{t+s}^{\hat{\gamma}}\left(F^{\theta}\right)=\int_{\widehat{\Gamma}_{*}} p_{t}^{\hat{\gamma}^{\prime}}\left(F^{\theta}\right) p_{s}^{\hat{\gamma}}\left(d \hat{\gamma}^{\prime}\right), \quad t, s \geq 0, \quad \theta \in \Theta
$$

By (2.25) we have

$$
\begin{aligned}
p_{t}^{\hat{\gamma}^{\prime}}\left(F^{\theta}\right) & =\exp \left(\int_{X} \int_{0}^{t} \theta_{\alpha}(x, 0) d \alpha \chi(d x)\right) \delta_{\hat{\gamma}^{\prime}}^{t}\left(F^{\theta}\right) \\
& =\exp \left(\int_{X} \int_{0}^{t} \theta_{\alpha}(x, 0) d \alpha \chi(d x)\right) F^{\theta_{t}}\left(\hat{\gamma}^{\prime}\right),
\end{aligned}
$$

see also (4.1) and (3.1). By the latter formula and (4.2), (4.4) we then get

$$
\begin{aligned}
\operatorname{RHS}(4.5) & =\exp \left(\int_{X} \int_{0}^{t} \theta_{\alpha}(x, 0) d \alpha \chi(d x)+\int_{X} \int_{0}^{s} \theta_{t+\alpha}(x, 0) d \alpha \chi(d x)\right) \delta_{\hat{\gamma}}^{s}\left(F^{\theta_{t}}\right) \\
& =\exp \left(\int_{X} \int_{0}^{t+s} \theta_{\alpha}(x, 0) d \alpha \chi(d x)\right) F^{\theta_{t+s}}(\hat{\gamma})=\operatorname{LHS}(4.5) .
\end{aligned}
$$

As is known, cf. Ethier and Kurtz (1986), Theorem 1.2, page 157, the transition function (4.4) determines a Markov process, $\mathcal{X}$, with values in $\widehat{\Gamma}$, the finite-dimensional distributions of which are given by the following formula

$$
\begin{aligned}
P\left(\mathcal{X}\left(s_{1}\right)\right. & \left.\in B_{1}, \ldots, \mathcal{X}\left(s_{n}\right) \in B_{n}\right) \\
& =\int_{\widehat{\Gamma}} \int_{B_{1}} \cdots \int_{B_{n-1}} p_{s_{n}-s_{n-1}}^{\hat{\gamma}_{n-1}}\left(B_{n}\right) p_{s_{n-1}-s_{n-2}}^{\hat{\gamma}_{n-2}}\left(d \hat{\gamma}_{n-1}\right) \times \cdots \times p_{s_{2}-s_{1}}^{\hat{\gamma}_{1}}\left(d \hat{\gamma}_{2}\right) p_{s_{1}}^{\hat{\gamma}_{1}}\left(d \hat{\gamma}_{1}\right) \mu(d \hat{\gamma}),
\end{aligned}
$$

holding for all $n \in \mathbb{N}, 0<s_{1} \leq s_{2} \leq \cdots \leq s_{n}$ and $B_{i} \in \mathcal{B}(\widehat{\Gamma})$. Here $\mu \in \mathcal{P}(\widehat{\Gamma})$ is the initial distribution of $\mathcal{X}$. Our aim is to show that such a process is unique up to modifications.

4.2. The statement. The process determined by (4.6) describes the stochastic evolution of the population which we consider. To verify whether it is the only one, we have to specify which processes of this kind can be associated to the model defined by the Kolmogorov operator (1.2). As is standard, the corresponding specification is made by their martingale property, see Ethier and Kurtz (1986), Chapt. 4.

Definition 4.1. Let $\mathcal{X}$ be a measurable process on some probability space $(\Omega, \mathfrak{F}, P)$ with values in $\widehat{\Gamma}$. Let also $\left\{\mathfrak{F}_{t}\right\}_{t \geq 0}$ be a filtration such that $\mathcal{X}(t)$ and

$$
\int_{0}^{t} G(\mathcal{X}(u)) d u
$$

are $\mathfrak{F}_{t}$-measurable for all $t$ and $G \in B(\widehat{\Gamma})$. We say that $\mathcal{X}$ is a solution of the martingale problem for $(L, \mathcal{D}(L))$ if for each $F \in \mathcal{D}(L)$,

$$
\mathcal{M}(t):=F(\mathcal{X}(t))-\int_{0}^{t}(L F)(\mathcal{X}(u)) d u
$$


is a $\mathfrak{F}_{t}$-martingale. If there exists a solution of the martingale problem for $(L, \mathcal{D}(L))$ and uniqueness holds, we say that the problem is well-posed. In the same way, we define the martingale problem for $(L, \mathcal{D}(L), \mu)$ if the initial distribution $\mu \in \mathcal{P}(\widehat{\Gamma})$ is specified.

The process related to the transition function (4.4) solves the martingale problem for $(L, \mathcal{D}(L))$. Its uniqueness will be shown by proving that all other solutions have the same finite-dimensional marginals, i.e., they coincide with those defined in (4.6). We are going also to show that the solution is temporarily ergodic.

Definition 4.2. Let the martingale problem for $(L, \mathcal{D}(L), \mu)$ be well-posed. Then $\mu \in \mathcal{P}(\widehat{\Gamma})$ is said to be a stationary distribution if for each $n$ and $0<s_{1}<s_{2}<\cdots<s_{n}$, the $n$-dimensional marginals introduced in (4.6) corresponding to $t+s_{1}, \ldots, t+s_{n}$ are independent of $t \geq 0$.

If $P$ is as in (4.6), then $\mu$ is stationary if and only if

$$
\mu=\int_{\widehat{\Gamma}} p_{s}^{\hat{\gamma}} \mu(d \hat{\gamma})
$$

holding for all $t>0$. Now we can formulate our result.

Theorem 4.3. The martingale problem for $(L, \mathcal{D}(L))$ is well-posed in the sense of Definition 4.1. Its solution is defined by finite-dimensional marginals, see (4.6), with the transition function defined in (4.4). If the departure function satisfies $m(\hat{x}) \geq m_{0}>0$, holding for all $\hat{x}$ and some $m_{0}$, then there exists a unique stationary distribution $\mu=\pi_{\varrho}$, which is the Poisson measure with intensity measure

$$
\varrho(d \hat{x})=\exp (-M(\hat{x})) \chi(d x) d \alpha .
$$

Moreover, in this case the solution $\mathcal{X}$ of the martingale problem for $(L, \mathcal{D}(L), \mu)$ is temporarily ergodic in the following sense. Let $\mu_{t} \in \mathcal{P}(\widehat{\Gamma})$ be the law of $\mathcal{X}(t), t \geq 0$. Then $\mu_{t} \Rightarrow \pi_{\varrho}$ as $t \rightarrow+\infty$.

\section{The Proof}

The proof of Theorem 4.3 is divided into the following steps:

(a) Proving uniqueness.

(b) Showing the stationarity and ergodicity if $m(\hat{x}) \geq m_{*}>0$.

The realization of (a) is based on the Fokker-Planck equation for $L$, which is a weak version of the forward Kolmogorov equation.

5.1. The Fokker-Planck equation. The Fokker-Planck equation is in a sense dual to the Kolmogorov equation (1.1), see Bogachev et al. (2015) for a general results on such equations. It describes the evolution of states of our model and reads

$$
\mu_{t}(F)=\mu_{0}(F)+\int_{0}^{t} \mu_{s}(L F) d s, \quad \mu_{0} \in \mathcal{P}(\widehat{\Gamma}) .
$$

Definition 5.1. By a solution of (5.1) we understand a map $\mathbb{R}_{+} \ni t \mapsto \mu_{t} \in \mathcal{P}(\widehat{\Gamma})$ possessing the following properties: (a) for each $F \in C_{\mathrm{b}}(\widehat{\Gamma})$, the map $\mathbb{R}_{+} \ni t \mapsto \mu_{t}(F) \in \mathbb{R}$ is measurable; (b) the equality in (5.1) holds for all $F \in \mathcal{D}(L)$.

Note that, for each solution $\mu_{t}$ and $F \in \mathcal{D}(L)$, the map $\mathbb{R}_{+} \ni t \mapsto \mu_{t}(F)$ is Lipschitz-continuous. Hence, it is almost everywhere differentiable, and its derivative is $\mu_{t}(L F)$. Therefore

$$
\mu_{t_{2}}(F)=\mu_{t_{1}}(F)+\int_{t_{1}}^{t_{2}} \mu_{s}(L F) d s, \quad 0 \leq t_{1}<t_{2} .
$$

It turns out that, for our model, one can construct solutions of (5.1) explicitly, which we are going to realize now. 
Lemma 5.2. For each $\mu_{0} \in \mathcal{P}(\widehat{\Gamma})$, the map $t \mapsto \mu_{t}$ defined in (4.3) is a unique solution of (5.1).

Proof: For each $\theta \in \Theta$, the map $t \mapsto \mu^{t}\left(F^{\theta}\right)=\mu_{0}\left(F^{\theta_{t}}\right)$ is continuous (by the dominated convergence theorem) and hence measurable. By (2.26) we then have

$$
\begin{aligned}
\mu_{t}\left(F^{\theta}\right) & =\pi_{t}\left(F^{\theta}\right) \mu^{t}\left(F^{\theta}\right)=\pi_{t}\left(F^{\theta}\right) \mu_{0}\left(F^{\theta_{t}}\right) \\
& =\exp \left(\int_{0}^{t}\left[\int_{X} \theta(x, \alpha) e^{-M(x, \alpha)} \chi(d x)\right] d \alpha\right) \mu_{0}\left(F^{\theta_{t}}\right) .
\end{aligned}
$$

Thus, the map $t \mapsto \mu_{t}\left(F^{\theta}\right)$ is continuous and hence measurable. Then the measurability of $t \mapsto \mu_{t}(F)$ for all $F \in C_{\mathrm{b}}(\widehat{\Gamma})$ follows by claim (ii) of Proposition 2.9. Now we turn to proving the equality in (5.1) for $F=F_{\lambda, \theta}, \theta \in \Theta$, see Definition 3.6. By (4.3) and (3.6) for $s, t \geq 0$ and $\theta \in \Theta$, we have

$$
\begin{aligned}
\mu_{t}\left(F_{s}^{\theta}\right) & =\exp \left(\int_{0}^{s}\left(\int_{X} \theta_{\alpha}(x, 0) \chi(d x)\right) d \alpha\right) \pi_{t}\left(F^{\theta_{s}}\right) \mu^{t}\left(F^{\theta_{s}}\right) \\
& =\exp \left(\int_{0}^{s}\left(\int_{X} \theta_{\alpha}(x, 0) \chi(d x)\right) d \alpha+\int_{0}^{t}\left(\int_{X} \theta_{s+\alpha}(x, 0) \chi(d x)\right) d \alpha\right) \mu_{0}\left(F^{\theta_{s+t}}\right) \\
& =\exp \left(\int_{0}^{s+t}\left(\int_{X} \theta_{\alpha}(x, 0) \chi(d x)\right) d \alpha\right) \mu_{0}\left(F^{\theta_{s+t}}\right)=\mu_{0}\left(F_{s+t}^{\theta}\right) .
\end{aligned}
$$

Then by Fubini's theorem and the latter fact we get

$$
\begin{aligned}
\mu_{t}\left(F_{\lambda, \theta}\right)-\mu_{0}\left(F_{\lambda, \theta}\right) & =\int_{0}^{+\infty} e^{-\lambda s}\left[\mu_{t}\left(F_{s}^{\theta}\right)-\mu_{0}\left(F_{s}^{\theta}\right)\right] d s \\
& =\int_{0}^{+\infty} e^{-\lambda s} \mu_{0}\left(F_{t+s}^{\theta}-F_{s}^{\theta}\right) d s=\int_{0}^{+\infty} e^{-\lambda s} \int_{0}^{t} \frac{\partial}{\partial u} \mu_{0}\left(F_{s+u}^{\theta}\right) d s d u \\
& =\int_{0}^{+\infty} e^{-\lambda s} \int_{0}^{t} \frac{\partial}{\partial s} \mu_{u}\left(F_{s}^{\theta}\right) d s d u=\int_{0}^{t} \mu_{u}\left(\int_{0}^{+\infty} e^{-\lambda s} \frac{\partial}{\partial s} F_{s}^{\theta} d s\right) d u \\
& =\int_{0}^{t} \mu_{u}\left(L F_{\lambda, \theta}\right) d u,
\end{aligned}
$$

where we have used also (3.41). Now we prove uniqueness by applying arguments similar to those used in Costantini and Kurtz (2015), Lemma 2.11. Assume that a map $t \mapsto \mu_{t}$ satisfies condition (a) Definition 5.1 and $F, G \in C_{\mathrm{b}}(\widehat{\Gamma})$ are such that

$$
\mu_{t}(F)-\mu_{0}(F)=\int_{0}^{t} \mu_{s}(G) d s
$$

holding for all $t \geq 0$. Then the map $t \mapsto \mu_{t}(F)$ is almost everywhere differentiable and

$$
d \mu_{t}(F)=\mu_{t}(G) d t .
$$

Then integrating by parts we get

$$
-\lambda \int_{0}^{t} e^{-\lambda s} \mu_{s}(F) d s=e^{-\lambda t} \mu_{t}(F)-\mu_{0}(F)-\int_{0}^{t} e^{-\lambda s} \mu_{s}(G) d s,
$$

which yields

$$
\mu_{0}(F)=e^{-\lambda t} \mu_{t}(F)+\int_{0}^{t} e^{-\lambda s}\left[\lambda \mu_{s}(F)-\mu_{s}(G)\right] d s
$$


holding for all $t, \lambda>0$. Passing here to the limit $t \rightarrow+\infty$, for $F=F_{\lambda, \theta}$ and $G=L F_{\lambda, \theta}$, see (5.1), we arrive at

$$
\mu_{0}\left(F_{\lambda, \theta}\right)=\int_{0}^{+\infty} e^{-\lambda s} \mu_{s}\left(\lambda F_{\lambda, \theta}-L F_{\lambda, \theta}\right) d s=\int_{0}^{+\infty} e^{-\lambda s} \mu_{s}\left(F^{\theta}\right) d s
$$

see (3.40). Assume now that (5.1) has two solutions, $\mu_{t}$ and $\tilde{\mu}_{t}$, satisfying the same initial condition $\left.\mu_{t}\right|_{t=0}=\left.\tilde{\mu}_{t}\right|_{t=0}=\mu_{0}$. By (5.2) the Laplace transforms of both maps $t \mapsto \mu_{t}\left(F^{\theta}\right)$ and $t \mapsto \tilde{\mu}_{t}\left(F^{\theta}\right)$ coincide, which yields $\mu_{t}\left(F^{\theta}\right)=\tilde{\mu}_{t}\left(F^{\theta}\right)$ holding for each $t$ and all $F^{\theta}, \theta \in \Theta$. Then the uniqueness in question follows by Proposition 2.9. This completes the whole proof.

5.2. Completing the proof. The existence of a solution of the martingale problem for $(L, \mathcal{D}(L))$ was shown by the very construction of the finite-dimensional marginals of $\mathcal{X}$ in (4.6). To prove uniqueness we use the following fact, see Ethier and Kurtz (1986), Proposition 4.2, page 184. Given $\mu \in \mathcal{P}(\widehat{\Gamma})$, let $\mathcal{X}$ and $\mathcal{X}^{\prime}$ be solutions of the martingale problem for $(L, \mathcal{D}(L), \mu)$ whose onedimensional marginals, $\mu_{t}$ and $\mu_{t}^{\prime}$, coincide for all $t \geq 0$. Then all their finite-dimensional marginals coincide and hence the problem is well-posed. Clearly, both $\mu_{t}$ and $\mu_{t}^{\prime}$ solve the Fokker-Planck equation with the initial condition $\mu$. Then they coincide by Lemma 5.2. This yields well-posedness.

Now we show the stated ergodicity. If $m$ satisfies $m(\hat{x}) \geq m_{0}>0$, then $M(x, \alpha) \geq m_{0} \alpha$, see (3.1), which for $\theta$ as in (3.1) yields

$$
\forall t>0 \quad\left|\theta_{t}(\hat{x})\right| \leq e^{-t m_{0}} .
$$

By the continuity of the map $t \mapsto \mu\left(F^{\theta_{t}}\right)$ we then get

$$
\begin{aligned}
\mu_{t}\left(F^{\theta}\right) & =\exp \left(\int_{0}^{t}\left[\theta_{\alpha}(x, 0) \chi(d x)\right] d \alpha\right) \mu\left(F^{\theta_{t}}\right) \\
& \rightarrow \exp \left(\int_{0}^{+\infty}\left[\int_{X} \theta_{\alpha}(x, 0) \chi(d x)\right] d \alpha\right), \quad t \rightarrow+\infty .
\end{aligned}
$$

By claim (iv) of Proposition 2.9 this yields $\mu_{t} \Rightarrow \pi_{\varrho}$, see $(4.7)$, holding for each initial $\mu \in \mathcal{P}(\widehat{\Gamma})$. Clearly, $\mu_{t}=\pi_{\varrho}$ if $\mu=\pi_{\varrho}$, which means that $\pi_{\varrho}$ is a stationary state. If there exists another stationary state, say $\mu^{\prime}$, then (5.3) fails to hold for $\mu=\mu^{\prime}$, which contradicts the convergence just established. This completes the proof of Theorem 4.3.

\section{Acknowledgements}

The research of both authors was financially supported by National Science Centre, Poland, grant 2017/25/B/ST1/00051. The second named author was also supported by the Deutsche Forschungsgemeinschaft through SFB 1238 "Taming uncertainty and profiting from randomness and low regularity in analysis, statistics and their applications". These supports are cordially acknowledged by the authors.

\section{Appendix}

Here we prove that $\tilde{r}$ introduced in (2.1) satisfies (2.2). During the whole proof, we deal with the function $\ell(\alpha)=\alpha+1 / \alpha \geq 2=\ell(1), \alpha>0$, which is decreasing for $\alpha \leq 1$ and increasing for $\alpha \geq 1$.

First, consider the case of $\underline{\alpha_{2}=0}$, where $\tilde{r}\left(\alpha_{1}, \alpha_{2}\right)=\omega\left(\alpha_{1}\right)$. For $\alpha_{i} \leq 1, i=1,3$, it follows that $\omega\left(\alpha_{i}\right)=\alpha_{i}$ and $\tilde{r}\left(\alpha_{1}, \alpha_{3}\right)=\left|\overline{\alpha_{3}-\alpha_{1}}\right|$. Hence, (2.2) turns into

$$
\alpha_{1} \leq\left|\alpha_{3}-\alpha_{1}\right|+\alpha_{3},
$$


which obviously holds true. For $\alpha_{3}>1$, we have the following two possibilities: (a) $\alpha_{3}-\alpha_{1} \leq$ $\alpha_{1}+1 / \alpha_{3}$; (b) $\alpha_{3}-\alpha_{1}>\alpha_{1}+1 / \alpha_{3}$. In (a), we get

$$
\omega\left(\alpha_{1}\right)=\alpha_{1} \leq \alpha_{3}-\alpha_{1}+1 / \alpha_{3}=\tilde{r}\left(\alpha_{1}, \alpha_{3}\right)+\omega\left(\alpha_{3}\right),
$$

which yields the inequality in question for this case since $2 \alpha_{1} \leq \ell\left(\alpha_{3}\right)$. Note that the equality in (.4) is possible only if $\alpha_{1}=\alpha_{3}=1$. In (b), we have $\alpha_{1} \leq \alpha_{1}+2 / \alpha_{3}$, which completes the proof of (2.2) for this case. For $\alpha_{1}>1$ and $\alpha_{3} \leq 1$, we have the following possibilities: (a) $\alpha_{1}-\alpha_{3} \leq \alpha_{3}+1 / \alpha_{1}$; (b) $\alpha_{1}-\alpha_{3}>\alpha_{3}+1 / \alpha_{1}$. In (a), we have

$$
\omega\left(\alpha_{1}\right)=1 / \alpha_{1} \leq \alpha_{1}=\alpha_{1}-\alpha_{3}+\alpha_{3},
$$

which yields (2.2) in this case. In case (b), we have

$$
\omega\left(\alpha_{1}\right)=1 / \alpha_{1} \leq \alpha_{3}+1 / \alpha_{1}+\alpha_{3}
$$

It remains to consider the cases: (i) $\alpha_{1} \geq \alpha_{3}>1$; (ii) $1<\alpha_{1}<\alpha_{3}$. For $\alpha_{1}-\alpha_{3} \leq 1 / \alpha_{1}+1 / \alpha_{3}$, in (i) we have

$$
\omega\left(\alpha_{1}\right)=1 / \alpha_{1} \leq 1 / \alpha_{3} \leq \alpha_{1}-\alpha_{3}+1 / \alpha_{3}
$$

which proves (2.2) for this case. For $\alpha_{1}-\alpha_{3}>1 / \alpha_{1}+1 / \alpha_{3}$, in (i) we have

$$
\omega\left(\alpha_{1}\right)=1 / \alpha_{1} \leq 1 / \alpha_{1}+1 / \alpha_{3}+1 / \alpha_{3},
$$

which again yields (2.2). For $\alpha_{3}-\alpha_{1} \leq 1 / \alpha_{1}+1 / \alpha_{3}$, in (ii) we have to prove $1 / \alpha_{1} \leq \alpha_{3}-\alpha_{1}+1 / \alpha_{3}$, which is equivalent to $\ell\left(\alpha_{1}\right) \leq \ell\left(\alpha_{3}\right)$. The latter follows by $\alpha_{3} \geq \alpha_{1}>1$ as $\ell$ is increasing. For $\alpha_{3}-\alpha_{1}>1 / \alpha_{1}+1 / \alpha_{3}$, the proof of (2.2) $\mathrm{f}$ is immediate.

Now we consider the case $0<\alpha_{1}<\alpha_{2}$. If $\alpha_{2} \leq 1$ and $\alpha_{3}=0$, then $\alpha_{2}-\alpha_{1} \leq \omega\left(\alpha_{1}\right)+\omega\left(\alpha_{2}\right)=\alpha_{1}+$ $\alpha_{2}$. For $\alpha_{3} \in(0,1]$, it follows that $\tilde{r}\left(\alpha_{3}, \alpha_{i}\right)=\left|\alpha_{3}-\alpha_{i}\right|$. Then (2.2) turns into the triangle inequality for $|\cdot|$. The same is true also for $\alpha_{3}>1$ such that $\alpha_{3}-1 / \alpha_{3} \leq 2 \alpha_{1}$. For $2 \alpha_{1}<\alpha_{3}-1 / \alpha_{3} \leq 2 \alpha_{2}$, the right-hand side of (2.2) is $\alpha_{3}-\alpha_{2}+\alpha_{1}+1 / \alpha_{3}$. Then $\alpha_{2}-\alpha_{1} \leq \operatorname{RHS}(2.2)$ turns into $2\left(\alpha_{2}-\alpha_{1}\right) \leq \ell\left(\alpha_{3}\right)$, which holds since $2\left(\alpha_{2}-\alpha_{1}\right) \leq 2<\ell\left(\alpha_{3}\right)$ for $\alpha_{3}>1$. For $2 \alpha_{2}<\alpha_{3}-1 / \alpha_{3}$, the right-hand side of (2.2) is $\alpha_{1}+\alpha_{2}+2 / \alpha_{3}$, which is bigger than $\alpha_{2}-\alpha_{1}$.

Consider now $0<\alpha_{1} \leq 1<\alpha_{2}$ and $\alpha_{2}-1 / \alpha_{2} \leq 2 \alpha_{1}$. The latter means that $\tilde{r}\left(\alpha_{2}, \alpha_{1}\right)=\alpha_{2}-\alpha_{1}$. For $\alpha_{3}=0$, the right-hand side of $\left(2 . \overline{2)}\right.$ is $\alpha_{1}+1 / \alpha_{2}$, and the latter turns into $\alpha_{2}-1 / \alpha_{2} \leq 2 \alpha_{1}$, which holds in this case. The same is true for $2 \alpha_{3} \leq \alpha_{2}-1 / \alpha_{2}$. For $\alpha_{2}-1 / \alpha_{2} \leq 2 \alpha_{3} \leq 2 \alpha_{1}$, the right-hand side of (2.2) is $\alpha_{2}-\alpha_{3}+\alpha_{1}-\alpha_{3}$, which is bigger than $\alpha_{2}-\alpha_{1}$ as $\alpha_{3} \leq \alpha_{1}$. For $\alpha_{1} \leq \alpha_{3} \leq 1$, the right-hand side of (2.2) is $\alpha_{2}-\alpha_{3}+\alpha_{3}-\alpha_{1}=\operatorname{LHS}(2.2)$, Next, consider $1<\alpha_{3} \leq \alpha_{2}$, where $\alpha_{3}-1 / \alpha_{3} \leq \alpha_{2}-1 / \alpha_{2} \leq 2 \alpha_{1}$. For $\alpha_{2}-1 / \alpha_{2} \leq \ell\left(\alpha_{3}\right)$, the right-hand side of (2.2) is $\alpha_{2}-\alpha_{3}+\alpha_{3}-\alpha_{1}=\operatorname{RHS}(2.2)$. The case of $\alpha_{2}-1 / \alpha_{2}>\ell\left(\alpha_{3}\right)>2$ is impossible since $\alpha_{2}-1 / \alpha_{2} \leq 2 \alpha_{1} \leq 2$. For $\alpha_{3}>\alpha_{2}$ such that $\alpha_{3}-1 / \alpha_{3} \leq 2 \alpha_{1}$ and $\alpha_{3}-1 / \alpha_{3} \leq \ell\left(\alpha_{2}\right)$, we have $\tilde{r}\left(\alpha_{3}, \alpha_{1}\right)=\alpha_{3}-\alpha_{1}$ and $\tilde{r}\left(\alpha_{3}, \alpha_{2}\right)=\alpha_{3}-\alpha_{2}$. Then the right-hand side of (2.2) is $\alpha_{3}-\alpha_{1}+\alpha_{3}-\alpha_{2}=2 \alpha_{3}-\left(\alpha_{2}+\alpha_{1}\right)$, which is bigger than $\alpha_{2}-\alpha_{1}$. The case of $\alpha_{3}-1 / \alpha_{3}>\ell\left(\alpha_{2}\right)$ is impossible for $\alpha_{3}-1 / \alpha_{3} \leq 2 \alpha_{1}$. For $\alpha_{3}>\alpha_{2}$ such that $\alpha_{3}-1 / \alpha_{3}>2 \alpha_{1}$ and $\alpha_{3}-1 / \alpha_{3} \leq \ell\left(\alpha_{2}\right)$, we have $\tilde{r}\left(\alpha_{3}, \alpha_{1}\right)=\alpha_{1}+1 / \alpha_{3}$ and $\tilde{r}\left(\alpha_{3}, \alpha_{2}\right)=\alpha_{3}-\alpha_{2}$. Then the right-hand side of (2.2) is $\ell\left(\alpha_{3}\right)-\alpha_{2}+\alpha_{1}$, which yields (2.2) in the form $2\left(\alpha_{2}-\alpha_{1}\right) \leq \ell\left(\alpha_{3}\right)$. By $\alpha_{2}-1 / \alpha_{2} \leq 2 \alpha_{1}, \alpha_{2}>1$, we have that $2\left(\alpha_{2}-\alpha_{1}\right) \leq 2 \sqrt{1+\alpha_{1}^{2}}$, whereas $\alpha_{3}-1 / \alpha_{3}>2 \alpha_{1}$ yields $\ell\left(\alpha_{3}\right)>\ell\left(\alpha_{*}\right)=2 \sqrt{1+\alpha_{1}^{2}}$, which proves (2.2) in this case. Here $\alpha_{*}$ is the positive solution of $\alpha-1 / \alpha=2 \alpha_{1}$. For $\alpha_{3}>\alpha_{2}$ such that $\alpha_{3}-1 / \alpha_{3}>2 \alpha_{1}$ and $\alpha_{3}-1 / \alpha_{3}>\ell\left(\alpha_{2}\right)$, we have $\tilde{r}\left(\alpha_{3}, \alpha_{1}\right)=\alpha_{1}+1 / \alpha_{3}$ and $\tilde{r}\left(\alpha_{3}, \alpha_{2}\right)=1 / \alpha_{3}+1 / \alpha_{2}$. Then (2.2) turns to

$$
\alpha_{2}-\alpha_{1} \leq \alpha_{1}+2 / \alpha_{3}+1 / \alpha_{2}
$$

which holds as $\alpha_{2}-1 / \alpha_{2} \leq 2 \alpha_{1}$.

Consider now $0<\alpha_{1} \leq 1<\alpha_{2}$ and $\alpha_{2}-1 / \alpha_{2}>2 \alpha_{1}$. The latter means that $\tilde{r}\left(\alpha_{1}, \alpha_{2}\right)=\alpha_{1}+$

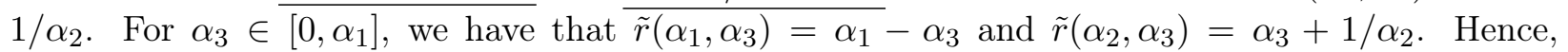
(2.2) turns into equality. For $\alpha_{3} \in\left(\alpha_{1}, 1\right]$ such that $\alpha_{2}-1 / \alpha_{2}>2 \alpha_{3}$, we have the right-hand 
side of (2.2) in the following form $\alpha_{3}-\alpha_{1}+\alpha_{3}+1 / \alpha_{2}$, which is bigger that $\tilde{r}\left(\alpha_{1}, \alpha_{2}\right)$ since $\alpha_{1}<$ $\alpha_{3}$. For $\alpha_{3} \in\left(\alpha_{1}, 1\right]$ such that $\alpha_{2}-1 / \alpha_{2} \leq 2 \alpha_{3}$, we have that the right-hand side of (2.2) is $\alpha_{3}-\alpha_{1}+\alpha_{2}-\alpha_{3}=\alpha_{2}-\alpha_{1}>\tilde{r}\left(\alpha_{1}, \alpha_{2}\right)$. Consider now $\alpha_{3}>1$ such that $\alpha_{3}-1 / \alpha_{3} \leq 2 \alpha_{1}$, which means that $\alpha_{3}<\alpha_{2}$ and $\tilde{r}\left(\alpha_{1}, \alpha_{3}\right)=\alpha_{3}-\alpha_{1}$. For $\tilde{r}\left(\alpha_{2}, \alpha_{3}\right)=\alpha_{2}-\alpha_{3}$, the right-hand side of (2.2) is $\alpha_{2}-\alpha_{3}+\alpha_{3}-\alpha_{1} \geq \tilde{r}\left(\alpha_{2}, \alpha_{3}\right)$. For $\tilde{r}\left(\alpha_{2}, \alpha_{3}\right)=1 / \alpha_{3}+1 / \alpha_{2}$, the right-hand side of (2.2) is $\alpha_{3}-\alpha_{1}+1 / \alpha_{2}+1 / \alpha_{3}$; hence, (2.2) turns into $2 \alpha_{1} \leq \ell\left(\alpha_{3}\right)$, which holds since $\alpha_{1} \leq 1$ and $\ell\left(\alpha_{3}\right) \geq 2$. For $\alpha_{3}>1$ such that $\alpha_{3}-1 / \alpha_{3}>2 \alpha_{1}$, we have that $\tilde{r}\left(\alpha_{1}, \alpha_{3}\right)=\alpha_{1}+1 / \alpha_{3}$. Then (2.2) turns into

$$
1 / \alpha_{2} \leq 1 / \alpha_{3}+\tilde{r}\left(\alpha_{2}, \alpha_{3}\right)
$$

which clearly holds for $\alpha_{3} \leq \alpha_{2}$, and also for $\alpha_{3}>\alpha_{2}$, where for $\tilde{r}\left(\alpha_{2}, \alpha_{3}\right)=\alpha_{3}-\alpha_{2}$ it turns into $\ell\left(\alpha_{2}\right) \leq \ell\left(\alpha_{3}\right)$ - which is true as $\ell(\alpha)$ is increasing for $\alpha>1$. For $\tilde{r}\left(\alpha_{2}, \alpha_{3}\right)=1 / \alpha_{3}+1 / \alpha_{2}$, the validity $(.5)$ is immediate.

Let us consider now the case of $\underline{1<\alpha_{1}<\alpha_{2}}$ and $\ell\left(\alpha_{1}\right) \leq \alpha_{2}-1 / \alpha_{2}$, where $\tilde{r}\left(\alpha_{2}, \alpha_{1}\right)=1 / \alpha_{1}+$ $1 / \alpha_{2}$. For $\alpha_{3} \leq 1$ such that $2 \alpha_{3} \leq \alpha_{1}-1 / \alpha_{1}$, we have $\tilde{r}\left(\alpha_{3}, \alpha_{i}\right)=\alpha_{3}+1 / \alpha_{i}, i=1,2$. Then (2.2) obviously holds. For $\alpha_{3} \leq 1$ satisfying $\alpha_{1}-1 / \alpha_{1}<2 \alpha_{3} \leq \alpha_{2}-1 / \alpha_{2}$, we have $\tilde{r}\left(\alpha_{3}, \alpha_{1}\right)=\alpha_{1}-\alpha_{3}$ and $\tilde{r}\left(\alpha_{3}, \alpha_{2}\right)=\alpha_{3}+1 / \alpha_{2}$. Hence, (2.2) turns into equality in this case. The remaining case $\alpha_{2}-1 / \alpha_{2}<2 \alpha_{3} \leq 2$ is impossible since $\alpha_{2}-1 / \alpha_{2} \geq \ell\left(\alpha_{1}\right)>2$. For $\alpha_{3}>1$ such that $2 \alpha_{3} \leq$ $\alpha_{1}-1 / \alpha_{1}$, we have $\tilde{r}\left(\alpha_{3}, \alpha_{i}\right)=1 / \alpha_{3}+1 / \alpha_{i}, i=1,2$. Then (2.2) obviously holds. For $\alpha_{3} \in\left(1, \alpha_{1}\right]$ satisfying $\alpha_{1}-1 / \alpha_{1}<2 \alpha_{3} \leq \alpha_{2}-1 / \alpha_{2},(2.2)$ turns into

$$
1 / \alpha_{1}+1 / \alpha_{2} \leq \alpha_{1}-\alpha_{3}+1 / \alpha_{2}+1 / \alpha_{3},
$$

which holds for $\alpha_{3} \leq \alpha_{1}$ as the function $\alpha-1 / \alpha$ is increasing. For $\alpha_{3} \in\left(\alpha_{1}, \alpha_{2}\right]$ such that $\alpha_{3}-1 / \alpha_{3} \leq$ $\ell\left(\alpha_{1}\right)<\ell\left(\alpha_{3}\right)<\alpha_{2}-1 / \alpha_{2}$, the right-hand side of (2.2) is $\alpha_{3}-\alpha_{1}+1 / \alpha_{3}+1 / \alpha_{2}$, and hence the latter turns into $\ell\left(\alpha_{1}\right)<\ell\left(\alpha_{3}\right)$. For $\alpha_{3} \in\left(\alpha_{1}, \alpha_{2}\right]$ satisfying $\alpha_{3}-1 / \alpha_{3} \leq \ell\left(\alpha_{1}\right)$ and $\alpha_{2}-1 / \alpha_{2} \leq \ell\left(\alpha_{3}\right)$, we have (2.2) in the form $1 / \alpha_{2}+1 / \alpha_{1} \leq \alpha_{2}-\alpha_{3}+\alpha_{3}-\alpha_{1}$, which holds as $\ell\left(\alpha_{1}\right)<\alpha_{2}-1 / \alpha_{2}$. For $\alpha_{3} \in\left(\alpha_{1}, \alpha_{2}\right]$ satisfying $\ell\left(\alpha_{1}\right)<\alpha_{3}-1 / \alpha_{3}$ and $\alpha_{2}-1 / \alpha_{2} \leq \ell\left(\alpha_{3}\right)$, the right-hand side of (2.2) is $1 / \alpha_{1}+1 / \alpha_{3}+\alpha_{2}-\alpha_{3}$ which is bigger than $\tilde{r}\left(\alpha_{1}, \alpha_{2}\right)$ as $\alpha-1 / \alpha$ is increasing. For $\alpha_{3}>\alpha_{2}$ such that $\alpha_{3}-1 / \alpha_{3} \leq \ell\left(\alpha_{2}\right)$, the right-hand side of (2.2) is $1 / \alpha_{1}+1 / \alpha_{3}+\alpha_{3}-\alpha_{2}$. Hence, (2.2) holds as $\ell\left(\alpha_{2}\right)<\ell\left(\alpha_{3}\right)$. For $\alpha_{3}-1 / \alpha_{3}>\ell\left(\alpha_{2}\right),(2.2)$ turns into $1 / \alpha_{1}+1 / \alpha_{2} \leq 1 / \alpha_{1}+1 / \alpha_{3}+1 / \alpha_{3}+1 / \alpha_{2}$.

Finally, let us consider the case of $\underline{1<\alpha_{1}<\alpha_{2}}$ and $\ell\left(\alpha_{1}\right)>\alpha_{2}-1 / \alpha_{2}$, where $\tilde{r}\left(\alpha_{2}, \alpha_{1}\right)=\alpha_{2}-\alpha_{1}$. For $\alpha_{3} \leq 1$ such that $2 \alpha_{3} \leq \alpha_{1}-1 / \alpha_{1}$, it follows that $\tilde{r} \overline{\left(\alpha_{i}, \alpha_{3}\right)=\alpha_{3}+1 / \alpha_{i}}, i=1,2$. Then (2.2) turns into $\alpha_{2}-1 / \alpha_{2} \leq \ell\left(\alpha_{1}\right)+2 \alpha_{3}$, which evidently holds in this case. For $\alpha_{1}-1 / \alpha_{1}<2 \alpha_{3} \leq \alpha_{2}-1 / \alpha_{2}$, we have that $\tilde{r}\left(\alpha_{1}, \alpha_{3}\right)=\alpha_{1}-\alpha_{3}$ and $\tilde{r}\left(\alpha_{2}, \alpha_{3}\right)=\alpha_{3}+1 / \alpha_{2}$. Then (2.2) turns into $\alpha_{2}-1 / \alpha_{2} \leq 2 \alpha_{1}$, which is the case for $2 \alpha_{1}>\ell\left(\alpha_{1}\right)>\alpha_{2}-1 / \alpha_{2}$. For $\alpha_{3} \leq 1$ satisfying $2 \alpha_{3}>\alpha_{2}-1 / \alpha_{2}$, it follows that $\tilde{r}\left(\alpha_{i}, \alpha_{3}\right)=\alpha_{i}-\alpha_{3}, i=1,2$. Then (2.2) turns into $2 \alpha_{3} \leq 2 \alpha_{1}$. which is obviously the case. For $\alpha_{3} \in\left(1, \alpha_{1}\right]$ such that $\ell\left(\alpha_{3}\right) \leq \alpha_{1}-1 / \alpha_{1}$, we have that $\tilde{r}\left(\alpha_{i}, \alpha_{3}\right)=1 / \alpha_{i}+1 / \alpha_{3}, i=1,2$. Then (2.2) amounts to $\alpha_{2}-1 / \alpha_{2} \leq \ell\left(\alpha_{1}\right)+2 / \alpha_{3}$, which obviously holds. For $\alpha_{1}-1 / \alpha_{1}<\ell\left(\alpha_{3}\right) \leq \alpha_{2}-1 / \alpha_{2}$, we have that $\tilde{r}\left(\alpha_{1}, \alpha_{3}\right)=\alpha_{1}-\alpha_{3}$ and $\tilde{r}\left(\alpha_{2}, \alpha_{3}\right)=1 / \alpha_{2}+1 / \alpha_{3}$. Then (2.2) amounts to $\alpha_{1}-\alpha_{3}+\alpha_{1}+$ $1 / \alpha_{3} \geq \alpha_{2}-1 / \alpha_{2}$, which is the case for $\alpha_{1}-\alpha_{3}+\alpha_{1}+1 / \alpha_{3} \geq \alpha_{1}+1 / \alpha_{3} \geq \ell\left(\alpha_{1}\right)>\alpha_{2}-1 / \alpha_{2}$. For $\alpha_{2}-1 / \alpha_{2}<\ell\left(\alpha_{3}\right)$, we have $\tilde{r}\left(\alpha_{i}, \alpha_{3}\right)=\alpha_{i}-\alpha_{3}, i=1,2$. Then (2.2) is $\alpha_{2}-\alpha_{1} \leq \alpha_{2}-\alpha_{3}+\alpha_{1}-\alpha_{3}$, which obviously holds as $\alpha_{3} \leq \alpha_{1}$. Now we consider $\alpha_{3} \in\left(\alpha_{1}, \alpha_{2}\right]$. For $\alpha_{3}-1 / \alpha_{3} \leq \ell\left(\alpha_{1}\right)$, we have $\tilde{r}\left(\alpha_{1}, \alpha_{3}\right)=\alpha_{3}-\alpha_{1}$ and $\tilde{r}\left(\alpha_{2}, \alpha_{3}\right)=\alpha_{2}-\alpha_{3}$, which yields equality in (2.2). Recall that $\alpha_{2}-1 / \alpha_{2}<\ell\left(\alpha_{1}\right)$; hence, $\alpha_{3}-1 / \alpha_{3}>\ell\left(\alpha_{1}\right)$ is impossible for $\alpha_{3} \leq \alpha_{2}$. It remains to consider $\alpha_{3}>\alpha_{2}$. For $\alpha_{3}-1 / \alpha_{3} \leq \ell\left(\alpha_{1}\right)$, we have that $\tilde{r}\left(\alpha_{i}, \alpha_{3}\right)=\alpha_{3}-\alpha_{i}, i=1,2$. Then (2.2) takes the form $\alpha_{2}-\alpha_{1} \leq 2 \alpha_{3}-\alpha_{1}-\alpha_{2}$, which obviously holds in this case. For $\ell\left(\alpha_{1}\right)<\alpha_{3}-1 / \alpha_{3} \leq \ell\left(\alpha_{2}\right),(2.2)$ amounts to $2 \alpha_{2} \leq \ell\left(\alpha_{3}\right)+\ell\left(\alpha_{1}\right)$, which holds as $\ell\left(\alpha_{1}\right)>\alpha_{2}-1 / \alpha_{2}$ (assumed) and $\ell\left(\alpha_{3}\right)>\alpha_{2}+1 / \alpha_{2}$ for $\alpha_{3}>\alpha_{2}$. For $\alpha_{3}-1 / \alpha_{3}>\ell\left(\alpha_{2}\right)$, we have that $\tilde{r}\left(\alpha_{3}, \alpha_{i}\right)=1 / \alpha_{3}+1 / \alpha_{i}, i=1,2$. Then (2.2) turns into

$$
\alpha_{2}-\alpha_{1} \leq 2 / \alpha_{3}+1 / \alpha_{2}+1 / \alpha_{1}
$$

which holds since $\ell\left(\alpha_{1}\right)>\alpha_{2}-1 / \alpha_{2}$. This completes the whole proof. 


\section{References}

Bogachev, V. I., Krylov, N. V., Röckner, M., and Shaposhnikov, S. V. Fokker-Planck-Kolmogorov equations, volume 207 of Mathematical Surveys and Monographs. American Mathematical Society, Providence, RI (2015). ISBN 978-1-4704-2558-6. MR3443169.

Costantini, C. and Kurtz, T. G. Viscosity methods giving uniqueness for martingale problems. Electron. J. Probab., 20, no. 67, 27 (2015). MR3361255.

Daley, D. J. and Vere-Jones, D. An introduction to the theory of point processes. Vol. II. General theory and structure. Probability and its Applications (New York). Springer, New York, second edition (2008). ISBN 978-0-387-21337-8. MR2371524.

Dawson, D. A. Measure-valued Markov processes. In École d'Été de Probabilités de SaintFlour XXI-1991, volume 1541 of Lecture Notes in Math., pp. 1-260. Springer, Berlin (1993). MR1242575.

Ethier, S. N. and Kurtz, T. G. Markov processes. Characterization and convergence. Wiley Series in Probability and Mathematical Statistics: Probability and Mathematical Statistics. John Wiley \& Sons, Inc., New York (1986). ISBN 0-471-08186-8. MR838085.

He, Z.-R., Ni, D., and Wang, S. Existence and stability of steady states for hierarchical agestructured population models. Electron. J. Differential Equations, pp. Paper No. 124, 14 (2019). MR4039327.

Jagers, P. and Klebaner, F. C. Population-size-dependent and age-dependent branching processes. Stochastic Process. Appl., 87 (2), 235-254 (2000). MR1757114.

Kozitsky, Y. Stochastic branching at the edge: individual-based modeling of tumor cell proliferation. J. Evol. Equ., 21 (2), 2081-2104 (2021). MR4278422.

Lenard, A. Correlation functions and the uniqueness of the state in classical statistical mechanics. Comm. Math. Phys., 30, 35-44 (1973). MR323270.

Méléard, S. and Tran, V. C. Slow and fast scales for superprocess limits of age-structured populations. Stochastic Process. Appl., 122 (1), 250-276 (2012). MR2860449.

Parthasarathy, K. R. Probability measures on metric spaces. Probability and Mathematical Statistics, No. 3. Academic Press, Inc., New York-London (1967). MR0226684.

Pazy, A. Semigroups of linear operators and applications to partial differential equations, volume 44 of Applied Mathematical Sciences. Springer-Verlag, New York (1983). ISBN 0-387-90845-5. MR710486.

Wang, Z. Stability and moment boundedness of an age-structured model with randomly-varying immigration or harvesting. J. Math. Anal. Appl., 471 (1-2), 423-447 (2019). MR3906332.

Zessin, H. The method of moments for random measures. Z. Wahrsch. Verw. Gebiete, 62 (3), 395-409 (1983). MR688646. 